\title{
IG NORK \\ Partner Choice, Investment in Children, and the Marital College Premium
}

\author{
Pierre-André Chiappori \\ Bernard Salanié \\ Yoram Weiss
}

Working Paper

2016-027

$11 / 2016$

HUMAN CAPITAL AND

ECONOMIC OPPORTUNITY

GLOBAL WORKING GROUP
The University of Chicago 1126 E. 59th Street Box 107 Chicago IL 60637 


\title{
Partner Choice, Investment in Children, and the Marital College Premium*
}

\author{
Pierre-André Chiappori ${ }^{\dagger} \quad$ Bernard Salanié ${ }^{\ddagger}$ \\ Yoram Weiss ${ }^{\S}$
}

November 16, 2016

\begin{abstract}
We construct a model of household decision-making in which agents consume a private and a public good, interpreted as children's welfare. Children's utility depend on their human capital, which is produced from parental time and human capital. We first show that as returns to human capital increase, couples at the top of the income distribution should spend more time on children. This in turn should reinforce assortative matching, in a sense we precisely define. We then embed the model into a Transferable Utility matching framework with random preferences à la Choo and Siow (2006) which we estimate on US marriage data for individuals born between 1943 and 1972. We find that the preference for assortative matching by education has significantly increased for the white population, particularly for highly educated individuals; but not for blacks. Moreover, in line with theoretical predictions, we find that the "marital college-plus premium" has increased for women but not for men.
\end{abstract}

\footnotetext{
*We thank Simon Lee, Steve Levitt, Corinne Low, Marcelo Moreira, Kevin Murphy, and seminar audiences in Amsterdam, Buffalo, Chicago, Delaware, CEMFI Madrid, LSE, Milan (Bocconi), Paris, Penn State, Rand Santa Monica, San Diego, Toronto and Vanderbilt for comments, as well as an editor and referees. We are also grateful to George-Levi Gayle, Limor Golan and Andres Hincapie for helping us generate Figure 13, and to Simon Weber for help with our code. Finally, we are deeply indebted to the late Gary Becker for his insightful comments. Financial support from the NSF (Grant SES-1124277) is gratefully acknowledged. So Yoon Ahn provided excellent research assistance.

†Columbia University.

$\ddagger$ Columbia University, corresponding author. Email: bsalanie@columbia.edu.

$\S$ Tel Aviv University.
} 


\section{Introduction}

Recent decades have been a period of significant demographic changes in the US and in other rich countries. The number of single-adult families has drastically grown, particularly at the bottom of the income distribution; moreover, several authors have argued that the degree of assortative matching among married couples has increased significantly ${ }^{1}$. Even more important are the evolutions in education and human capital, particularly in terms of composition by gender. During the first half of the twentieth century, college attendance increased for both genders, and slightly faster for men. According to Claudia Goldin and Larry Katz (2008), male and female college attendance rates were about $10 \%$ for the generation born in 1900, and reached respectively $55 \%$ and $50 \%$ for men and women born in 1950. This common trend, however, broke down for the cohorts born in the 1950s and later. This confronts economists with an interesting puzzle. Individuals born in these later decades experienced a rate of return on higher education in the labor market (the "college premium") that was substantially higher than their predecessors; therefore one would have expected their college attendance rate to keep increasing, possibly at a faster pace. This prediction is satisfied for women: $70 \%$ of the generation born in 1975 attended college. On the contrary, the male college attendance rate increased at a much slower rate, if at all. The phenomenon is especially striking for post-graduate education (the so-called "college-plus" levels: MAs, MBAs, law and medicine degrees, $\mathrm{PhD}$ ), for which the labor-market premium has risen even more. The fraction of women aged 30 to 40 with a college-plus degree rose from below $4 \%$ in 1980 to above $11 \%$ in 2005 , while the male proportion hardly changed over the same period. As a result, in recent cohorts women have been more educated than men, and by an increasing margin.

The main argument of the present paper is that these various demographic phenomena are the by-products of a general trend: the increasing importance of investment in education, particularly at the top of the distribution of human capital. As a result, the structure of household production has drastically changed; and so have motivations for marriage. To illustrate it, we construct a simple collective household model in which spouses consume two commodities, a private consumption good and a public good which we interpret as children's (future) utility. This public good is produced by parental time and human capital, using a Cobb-Douglas technology as in Daniela Del Boca, Christopher Flinn and Matthew Wiswall (2014). In addition, an exogenous amount of time must be devoted to basic chores (cleaning, cooking, etc.), activities for which spousal times are perfect substitutes. We show that the combination of technical progress in domestic technology (what Jeremy Greenwood et al., 2005, called

\footnotetext{
${ }^{1}$ This view is supported by a recent sociological literature that concludes that homogamy has increased in the US and several other countries (see for instance Schwartz and Mare, 2005). Burtless (1999) argues that this evolution complements the increase in the labor market college premium in explaining increased interhousehold income inequality. This vision has recently been emphasized by Greenwood et al (2014), who find that "if people matched in 2005 according to the 1960 standardized mating pattern there would be a significant reduction in income inequality; i.e., the Gini drops from 0.43 to 0.35 ." (p. 352).
} 
"engines of liberation") and a larger return on investment in children's human capital (in terms of the children's future income and ultimately well-being) has contrasted implications for time use: while less and less time should be spent on basic chores, time spent with children should increase, especially for high income/high education couples.

Following Becker's seminal contributions (see Gary Becker 1973, 1974, 1991), we next embed this household model into a frictionless matching framework with Transferable Utility (TU). From a theoretical perspective, this builds on and extends a recent contribution by Pierre-André Chiappori, Murat Iyigun and Yoram Weiss (2009, from now on CIW). Their paper argued that the strikingly genderasymmetric changes in the demand for higher education could be explained by recognizing that the returns to education comprise not only the standard labor market college premium, but also the effect of education on marital outcomes. Human capital affects not only future wages, but also the probability of getting married, the characteristics of the future spouse, and the size and distribution of the surplus generated within marriage. CIW advanced the hypothesis that, unlike the labor market premium which is largely gender-neutral, this "marital college (and college-plus) premium" may have evolved in a highly asymmetric way between genders.

We provide a microfoundation to this argument by showing that as parents' education is an important input in the production process of children human capital, matching should be assortative on human capital. If economic returns to investments in children's human capital increase, particularly at the top of the human capital distribution, then we should observe a corresponding increase in assortative matching, in a sense that we define precisely. Moreover, and in line with CIW, we show that such changes generate a positive feedback loop, as the new equilibrium returns further increase the incentives of (future) parents to invest in their own education. The intuition is that in addition to the standard labor market returns, the increase in assortative matching makes a higher stock of human capital even more valuable on the marriage market. Finally, as the importance of investment in children's human capital grows and as technological innovations reduce the time needed for domestic chores, the distribution of human capital changes within couples. The traditional pattern in which the man was typically more educated than the wife becomes less and less sustainable as the matching equilibrium generates an increased "marital higher education premium" for women. This is reflected in the asymmetric responses observed in the data. On this our analysis is in line with a recent contribution by Shelly Lundberg and Robert Pollak (2013), who emphasize a shift in the primary source of the gains to marriage, from the production of household services and commodities to investment in children ${ }^{2}$.

Establishing the empirical relevance of this theoretical argument is a chal-

\footnotetext{
${ }^{2}$ Mathias Doepke and Michelle Tertilt (2009) and Doepke, Tertilt and Alessandra Voena (2011) have analyzed a different but related phenomenon, with the regards the political economy of womenâs rights. In their model, an increase in the return to human capital induces men to vote for women's rights, which is turn promotes growth in human capital. While this mechanism is not explicit considered in our framework, it is compatible with it.
} 
lenging task. For one thing, the mere notion of "increased assortative matching" is not easy to measure. There is no doubt that the percentage of couples in which both spouses have a college or "college-plus" degree has significantly increased. However, this evolution at least partly reflects the shifts in the number of educated women; such a mechanical effect must be distinguished from possible changes in the preference for homogamy. A precise definition of "changes in the degree of assortative matching" has to disentangle these two effects; this is not an easy task. In addition, while the returns to schooling in the labor market can be recovered from observed wage data, neither the size of the surplus, nor the subsequent returns to schooling within marriage are directly observed; they can only be estimated indirectly from the marriage patterns of individuals with different levels of schooling.

A second contribution of our paper is to provide an empirical estimation of these various effects. We build upon a seminal contribution by Eugene Choo and Aloysius Siow (2006, from now on CS $)^{3}$. CS modelled the joint marital surplus as the sum of a systematic, deterministic component that only depends on observable traits (in our case education levels) and a stochastic part that reflects unobserved heterogeneity. They assumed that this stochastic part is the sum of a wife- and a husband-specific parts, each of which only depends on the education of the potential spouse. Combined with a well-chosen distributional assumption, this separability allowed them to translate the matching equilibrium conditions into a simple, discrete choice structure It is well known that the values of the utilities obtained by participants in equilibrium can be computed as multipliers in the maximization of aggregate surplus over all possible matchings. We show that in the CS framework, the stochastic distribution of these dual variables can be fully characterized. In particular, one can identify the distribution of expected utility for any possible choice of a spouse with a given education, as well as for the optimal choice; one can therefore compute the increase in expected utility resulting from a higher stock of human capital - a notion that is directly related to the concept of marital college premium. We also propose a precise definition of "preference for homogamy": in our framework, it is simply the supermodularity of the surplus along the diagonal.

More importantly, we extend the CS approach to a "multi-market" framework $^{4}$. This allows us both to relax the restrictive assumptions imposed by the initial CS contribution, to estimate changes over time, and to test overidentifying restrictions implied by the model. To do this, we assign successive cohorts to different marriage markets. The changing proportions of men and women at all education levels introduce exogenous variation that drives our identification. In particular, our model is compatible with education-specific distributions of

\footnotetext{
${ }^{3} \mathrm{CS}$ studied the response of the US marriage market to the legalization of abortion. See Maristella Botticini and Siow (2008) and Siow (2015) for other applications. Alfred Galichon and Salanié (2015) generalized the Choo and Siow framework to arbitrary separable stochastic distributions; they also provide a theoretical and econometric analysis of multicriterial matching under the same separability assumption. Isaac Mourifié and Siow (2014) extend the Choo and Siow model in another direction, by allowing for peer effects in the joint surplus.

${ }^{4}$ See Jeremy Fox (2010a, 2016) and Fox and David Hsu and Chenyu Yang (2015) for different approaches to pooling data from several markets.
} 
unobserved heterogeneity, thus allowing for selection into education being correlated with preferences for marriage - a natural consequence of $\mathrm{CIW}^{5}$. It can also incorporate class-specific temporal drifts in the systematic component of the surplus. These features allow us to study the evolution of matching patterns, and in particular changes in assortative matching, in a flexible context where both gains from marriage and the intra-household allocation of these gains change over time for each education class. The variation across cohorts makes it possible to disentangle variations in the surplus generated by assortative matching from the mechanical effects of the observed changes in distributions of male and female education. We model this by allowing the supermodular part of the surplus to evolve according to a linear, education-dependent time trend. These features, together with the emphasis we put on the micro foundations of our model (and their testable implications regarding household behavior), distinguish our work from a recent paper by Siow (2015) which finds that supermodularity has not increased in the US between 1970 and 2000. The model used by Siow is exactly identified in each year. This allows him to observe the evolution of supermodularity (and in principle to test that the resulting estimates are statistically different across years). But for lack of a more constrained framework such as ours, it does not allow to elucidate the nature of the changes. In contrast, our model is overidentified in a multi-market context. In particular, we can identify trends over time and test their significance.

The empirical results we obtain for the white population corroborate our model at two levels. First, time use data indicate that while time spent on basic chores significantly decreased for women over the period (while it slightly increased for men, a pattern predicted by theory), time spent on and with children increased sharply for both spouses. This result is all the more striking that female wages have increased over the period, raising their corresponding opportunity cost; but it is fully compatible with our explanation based on the large increase in the return on investment in children's education. Regarding the second set of theoretical predictions, we find strong evidence that the surplus generated by assortativeness ${ }^{6}$ has increased, particularly at the top of the education distribution. Finally, the evolution of marital returns to education is highly gender-specific: while they did not change much for men, they increased significantly for women.

The marriage patterns of the black population, however, present us with intriguing differences. It is well-documented that after World War II, the marriage patterns of whites and blacks started to diverge: the marriage rates of blacks fell faster for both genders. Starting with Wilson and Neckerman (1986), a growing literature has attributed these trends to the shortage of "marriageable" black men. We bring into this discussion differences across groups in preferences for assortative marriage by education, and the resulting differences

\footnotetext{
${ }^{5} \mathrm{CIW}$ implies a positive correlation between marital preferences and demand for education: individuals who are more eager to marry an educated spouse (especially) have an additional incentive to invest in their own education. Our framework, however, is agnostic on this point: any correlation between marital preferences and demand for education is possible a priori.

${ }^{6}$ What we call later the "supermodular core" of the surplus.
} 
in the marital college premium. We find no evidence of changes in the surplus generated by assortativeness among blacks: the supermodularity of the surplus function hardly changed over time. Moreover, the "marital college premium" evolved in pretty much the same way for men and women. These patterns are consistent with another finding of the recent empirical literature - namely, that black couples spend much less time with their children than whites ${ }^{7}$. Many other differences, of course, probably also come into play. There is definitely a need for further research on this topic.

Section 2 presents some stylized facts. Then we introduce our theoretical framework in Section 3, and section 4 describes the basic principles underlying its empirical implementation. Section 5 explains how we conduct estimation and testing. Our empirical findings are presented in Section 6 .

\section{The Data}

\subsection{Constructing the data}

We begin by describing our data and some stylized facts about the evolution of matching by education over the last decades in the US. We use the American Community Survey, a representative extract of the Census, which we downloaded from IPUMS (see Steven Ruggles et al 2015.) Since 2008 the survey has collected information on current marriage status, number of marriages, and year of current marriage. Our analysis uses the 21,583,529 households in the 2008 to 2014 waves. From this population, we extract all white and black adults (aged 18 or more) who are out of school. We use the "detailed education variable" of the ACS to define five subcategories:

1. High School Dropouts (HSD)

2. High School Graduates (HSG)

3. Some College (SC) — including two-year (associate) degrees

4. Four-year College Graduates (CG)

5. Graduate degrees ("college-plus", or CG+.)

However, the black population in our sample is smaller and less educated; therefore in our econometric analysis we will merge categories 4 and 5 into a single, "college and college-plus" category.

When empirically studying matching patterns, one has to address several practical issues. The first one is what constitutes "marriage". We treat as married households who define themselves as such; we do not attempt to include cohabitation with marriage. Cohabitation has grown over time, but it was rarer for the cohorts we consider (up to women born 1971). The National Survey of

\footnotetext{
${ }^{7}$ See for instance Georges-Levi Gayle et al (2015).
} 
Family Growth (NSFG) gives more information on marital/cohabitation histories; we looked more closely at individuals aged 40 or more in the 2011-13 waves of the survey. $20 \%$ of these women and one quarter of these men had never married. Among them, $26 \%$ of white women and $16 \%$ of white men were currently cohabiting; the corresponding numbers are 5\% and 18\% among blacks. Higher education individuals were less likely to cohabit; but this difference affects such a small proportion of the population (to fix ideas, $20 \%$ of $20 \%$ is $4 \%$ ) that it is very unlikely to have a significant impact on our findings. The distinction between marriage and cohabitation could in principle be analyzed within our framework. We tend to agree with Lundberg and Pollak (2013) that marriage entails an additional degree of commitment that is particularly helpful for longterm investments such as children education. Unfortunately, our data does not allow us to investigate this choice margin ${ }^{8}$.

Then we need to decide which matches to consider: the current match of a couple, or earlier unions in which the current partners entered? Also, do we define a single as someone who never married, or as someone who is currently not married? It is notoriously hard to model divorce and remarriage in an empirically credible manner ${ }^{9}$. Since this is not the object of this paper, we chose instead to only keep first unions, and never-married singles. Given this sample selection, in each cohort we miss those individuals who died before the survey; and we discard those who are single in the survey year but were married before, as well as those who married during the year in which they were surveyed.

We also discard "institutional households"; these correspond to correctional institutions, but also military and mental care facilities. We also do not know whether a given individual we observe in a normal household in 2010, say, was incarcerated when (s)he was younger. Since incarceration rates more than doubled, over the period, this is a serious concern for some subpopulations: at any point in time in the 1990s, a quarter of young black men without a high school diploma were incarcerated ${ }^{10}$. This is still probably better than the alternative, which would include institutionalized singles in the population available for marriage.

Another standard problem is truncation: young men and women who are single in the survey year may marry in future years. In our figures (and later in our estimates) we circumvent this difficulty by stopping at the male cohort born in 1968 (1963 for blacks); this choice is motivated by the fact that the first union occurs before age 40 ( 45 for blacks ${ }^{11}$ ) for most men and women. To examine marriage patterns, we also drop the small number of couples where one

\footnotetext{
${ }^{8}$ If one agrees with Lundberg and Pollak's view, then classifying cohabiting couples as singles (as we do) is probably an acceptable solution. Their argument indeed suggests that the decision not to marry is indicative - at least for the cohorts under consideration - of a lesser willingness to invest in children education, a key driver of marriage.

${ }^{9}$ Information on marital dissolution by education of the partners is available in the PSID, SIPP and NSFG but these samples are much smaller than the IPUMS sample that we use to analyze marriages.

${ }^{10}$ See Derek Neal and Armin Rick 2014.

${ }^{11}$ The data show that blacks continue to marry later than whites.
} 
partner married before age 16 or after age 40 (45 for blacks $)^{12}$.

Our final sample consists of

- $1,502,157$ white couples

- 78,759 black couples

- 542,677 white singles

- 136,052 black singles.

The sample of singles is slightly skewed towards males ( $52.8 \%$ vs $47.2 \%$ ). This conceals a large difference between races: males are only $39.5 \%$ of black singles, but they constitute $56.1 \%$ of white singles.

Finally, we need to define "cohorts". Ideally, each cohort should represent a "market" (or a matching game), involving specific populations; and we would relate changes in matching patterns to variations in the distribution of education by gender across cohorts as well as to changes in surplus. As always, reality is more complex, and the various "cohorts" tend to mix. For instance, if we define a cohort by the year of birth, then the spouse of a man born in 1957 is most likely to be born in 1958: the modal age difference is one year in our data. Yet such a man may well marry a woman born in 1956 or in 1960. Defining broader cohorts (e.g., men born between 1955 and 1960) does not solve the mixing problem; and it has the additional drawback that it reduces the number of cohorts. We have two approaches to this problem. Our benchmark model concentrates on couples in which the difference takes some fixed value (the modal value of one year in our case.) Then we consider men born between 1940 and 1967 and women born between 1941 and 1968, a total of 28 cohorts. We also impelment an extension that explicitly models the age difference as a choice parameter, the difference between husband's and wife's age as a choice parameter, which can take six values; in this case the cohorts become 1940-1967 for men and 1940-1971 for women. Reassuringly, the two approaches give similar results.

\subsection{Patterns in the data}

\subsubsection{White couples}

The trends in education levels of white men and women are shown in Figures 1 and 2 . In cohorts born after 1955, women are more likely than men to attend college; for those born after 1965, they are also more likely to achieve a college-plus degree. Not coincidentally, the proportion of marriages in which the husband is more educated than the wife has fallen quite dramatically. Indeed, Figure 2 shows that the percentage of couples in which spouses have the same education is remarkably stable (slightly below $50 \%$ ) over more than three decades. However, there are now more couples in which the wife is more educated than the opposite.

\footnotetext{
${ }^{12}$ Recall that these are first unions.
} 
[Figure 1 about here.]

[Figure 2 about here.]

Figure 3 illustrates the decline in marriage among whites by plotting the percentage of individuals who never married by cohort and education. They show that, for both genders, a higher education has tempered the decline in marriage. High-school dropouts, on the other hand, have faced a very steep decline in marriage rates. For the older cohorts of our sample, a college-plus degree had a strong, negative effect on the probability of getting married for women, but not for men. This gender difference has largely disappeared in recent cohorts: college-plus women now marry as much as college graduates, and much more than high-school educated women.

[Figure 3 about here.]

Figures 4 and 5 describe marital patterns by education. They show that college-educated men are now much less likely to "marry down" (about 25\%, against $50 \%$ for men born in the early 1940s). The pattern for women is opposite; for instance, the proportion of college-educated women who marry up (with a college-plus husband) has dropped from $40 \%$ to $20 \%$ over the period.

[Figure 4 about here.]

[Figure 5 about here.]

\subsubsection{Black couples}

[Figure 6 about here.]

[Figure 7 about here.]

[Figure 8 about here.]

The education and marriage patterns of blacks show some striking differences from whites. Black women have been at least as likely as men to get a college or a college-plus degree in every cohort. In contrast with the white population, the proportions of a cohort with at least a college degree evolve in a very similar way for men and for women (Figure 6.) As a consequence and in striking contrast to white couples, Figure 7 shows that in black couples of every cohort the wife is likely to be more educated than the husband; and this difference has increased in recent years.

Marriage rates have also declined much faster for blacks than for whites (see Figure 8.) For the cohorts born in the mid 1960s, the fraction of never married at age 45 is above $40 \%$ for all education classes, and exceeds $80 \%$ for high school drop-outs. Thirdly, for the older cohorts, one does not observe the same difference between genders as for whites. While the percentage of couples with equal education are similar for blacks and whites (a stable proportion around 
$45 \%$ ), in unequally educated couples the wife is always more likely to be the more educated person among blacks. Finally, the proportion of college educated individuals who never marry was much larger for men than women in the older cohorts; recent percentages are quite similar. In other words, the spectacular differences across genders that characterize the white population cannot be seen in the African-American sample ${ }^{13}$.

[Figure 9 about here.]

[Figure 10 about here.]

It is also worth noting that the marital patterns by education of black men and women who do marry are not that different from those of whites (compare Figures 9 and 10 to Figures 4 and 5.) The main difference is that fewer men "marry down" in the black population.

\subsubsection{Age differences}

Finally, the evolution of age differences between spouses for the white and black populations is shown in Figures 11 and 12, where "early" and "late" refer to the first and the last three cohorts respectively. The age difference has decreased slowly over time. Men are still more likely to be the older partner; but couples in which the woman is older are quite common in more recent cohorts $(29 \%$ of white couples and $33 \%$ of black couples are to the left of the dashed line in the rightmost panel.) The proportion of couples in which the (absolute) age difference is larger than ten years has become very small.

[Figure 11 about here.]

[Figure 12 about here.]

A large literature has evaluated the changes in the value of a higher education on the labor market. While this labor market college premium seems to have evolved in very similar ways across genders and races, the patterns documented here clearly suggest that the effects of a higher education on marital prospects have diverged much more. Yet descriptive statistics alone cannot measure changes in the joint surplus of matches and in its division between the partners; a more precise evaluation of this "marital college premium" requires an explicit structural model.

\footnotetext{
${ }^{13}$ We should also note here the increase in interracial marriages that started around 1960 and accelerated recently. Black men are much more likely than black women to marry a white spouse - see Gullickson (2006) and Cherlin (1992, Chapter 4.) Our model does not consider these additional dimensions, as interracial marriage was less frequent in our sample period.
} 


\section{Theoretical Framework}

Our model derives from CIW, who consider an economy with two periods and large numbers of men and women. In period one, agents draw costs of investment in human capital and marital preferences from some random distributions; then they invest in education by choosing from a finite set of possible educational levels. In period 2, agents match on a frictionless marriage market with transferable utility; they each receive a wage, the realization of which depends on the agent's education; and they consume according to an allocation of resources that was part of the matching agreement.

When investing in human capital, agents must anticipate the outcome of their investment. This outcome has two distinct components. One is a larger future wage. In our framework, this effect is taken to be exogenous, and to benefit single and married agents alike. Second, a higher educational level has an impact on marital prospects; it affects the probability of getting married, the expected income of the future spouse, the total utility generated within the household, and the intra-couple allocation of this utility. These marital gains, however, depend on the equilibrium reached on the marriage market; this in turn depends on the distribution of education in the two populations, and ultimately of the investment decisions made in the first period. As usual, the model can be solved backwards using a rational expectations assumption; equilibrium is reached when the marital gains resulting from given distributions of education for men and women trigger first period investment decisions that exactly generate these distributions. Note that even if marital preferences and investment cost were independent ex ante, education decisions made during the first period must be correlated with preferences for marriage ex post: since agents with stronger preferences for marriage are more likely to receive the marital gain than agents who prefer to stay single, they have stronger incentives to invest in education.

In the present paper, we aim at estimating and testing the second period behavior described by this model. This choice is mostly dictated by available data: while private costs of human capital investment are not observable, the resulting distribution of education by gender is. In addition, concentrating on the second period allows us to introduce a slightly more general framework while addressing the empirical content of the key theoretical concept: the notion of a marital college premium. We therefore consider the situation at the beginning of the second period. Agents are each characterized by their chosen level of education, which belongs to some finite set and is observable by all, and by their preferences for marriage, which are observed by their potential mates but not by the econometrician. Our goal is to identify the underlying structure from observed matching patterns; and we are particularly interested in the marital gains associated with each educational level. Lastly, and as explained in the introduction, we follow the literature ${ }^{14}$ by assuming that the utility of each agent is the sum of a deterministic component, which reflects the agent's consump-

\footnotetext{
${ }^{14}$ See for instance Chiappori and Salanié (2016) for a survey.
} 
tion and investment choices, and a shock that reflects the agent's idiosyncratic marital preferences.

\subsection{Preferences: the economic component}

The economy consists of a male population $\mathcal{M}$ and a female population $\mathcal{F}$, who only differ by their human capital. Education is the outcome of individual choices made before the matching stage. At the beginning of the matching game, these classes are therefore considered by the agents as exogenously given. However, the outcome of the matching game will, in equilibrium, impact the investment decision; this is the gist of CIW's contribution.

\subsubsection{Producing children's human capital}

In our model, the primary purpose of marriage is the production of a public good, namely children's (future) welfare. For simplicity, we assume that each couple has exactly one child. We are mostly interested here in human capital accumulation; therefore we assume that a child's future well being is a function of the child's human capital $Q$ :

$$
U_{C}=Q^{\alpha}
$$

where $\alpha$ is a parameter that summarizes the impact of human capital on the child's future wages, income dynamics, and ultimately welfare.

For simplicity, we assume that each couple has one child. The child's human capital is produced using parental time; other inputs could be introduced without changing the main conclusions. Following the recent literature on human capital production, we assume that the impact of parental time is proportional to the parent's own human capital, and that mother's and father's time inputs are complements in the production of the child's human capital. ${ }^{15}$ In practice, we use a Cobb-Douglas form:

$$
Q=\left(H_{1} t_{1}\right)^{\beta}\left(H_{2} t_{2}\right)^{1-\beta}
$$

where $H_{i}$ and $t_{i}$ respectively denote parent $i$ 's human capital and time spent with the child.

Alternatively, agents may use available time to work on the market or to accomplish domestic chores. On the labor market, an agent's wage is directly proportional to her human capital:

$$
w_{i}=W H_{i}
$$

where $W$ is some aggregate shock. We assume for simplicity that chores require some fixed amount of time $\tau^{\prime}$, for which male and female time are perfect substitutes; and that they involve no human capital. As a result, chores will be

\footnotetext{
${ }^{15}$ Recent work incorporating such complementarities includes del Boca, Flinn and Wiswall (2012).
} 
entirely performed by the spouse whose market wage is lower, whom we number as spouse 2. The spouses' respective time constraints are therefore:

$$
t_{1}+l_{1}=1 \text { and } t_{2}+l_{2}=\tau=1-\tau^{\prime}
$$

where $l_{i}$ denotes $i$ 's market work and $\tau$ is total time available for agent 2 .

\subsubsection{Preferences, surplus and individual welfare}

Continuous version Individual utilities consist of an economic and a non economic, predetermined component. The economic component depends on private and public consumptions according to a Cobb-Douglas form:

$$
u_{i}\left(q_{i}, U_{C}\right)=q_{i} U_{C}=q_{i} Q^{\alpha}
$$

where $q_{i}$ denotes $i$ 's private consumption. The couple's budget constraint is therefore:

$$
q_{1}+q_{2}=W H_{1}\left(1-t_{1}\right)+W H_{2}\left(\tau-t_{2}\right)
$$

or equivalently

$$
q_{1}+q_{2}+W H_{1} t_{1}+W H_{2} t_{2}=\left(H_{1}+\tau H_{2}\right) W
$$

These Cobb-Douglas preferences are of the Bergstrom and Cornes' generalized quasilinear form ${ }^{16}$; therefore they imply transferable utility. It follows that any efficient allocation maximizes the sum of utilities under the budget constraint:

$$
G\left(H_{1}, H_{2}\right)=\max \left(q_{1}+q_{2}\right) Q^{\alpha}
$$

under (1). The solution is:

$$
\begin{aligned}
H_{1} t_{1} & =\frac{\alpha \beta}{1+\alpha}\left(H_{1}+\tau H_{2}\right), \quad H_{2} t_{2}=\frac{\alpha(1-\beta)}{1+\alpha}\left(H_{1}+\tau H_{2}\right) \\
\text { and } q_{1}+q_{2} & =\frac{1}{1+\alpha}\left(H_{1}+\tau H_{2}\right) W .
\end{aligned}
$$

In particular, we see that both $t_{1}$ and $t_{2}$ are increasing in $\alpha$ and in $\tau$. That is, the larger the importance of children's human capital for their future welfare, the more time both parents will invest in producing this human capital. Moreover, a reduction in the time devoted to chores, by raising total available time $\tau$, will have the same impact. Also note that the value of the sum of utilities at the optimum is

$$
G\left(H_{1}, H_{2}\right)=K\left(H_{1}+\tau H_{2}\right)^{1+\alpha} W
$$

where

$$
K=\frac{\alpha^{\alpha} \beta^{\alpha \beta}(1-\beta)^{\alpha(1-\beta)}}{(1+\alpha)^{1+\alpha}}
$$

${ }^{16}$ See Ted Bergstrom and Richard Cornes (1983). 
Similarly, let $G^{i}\left(H_{i}\right), i=1,2$, denote the utility of person $i$ when single, so that the economic surplus generated by marriage is

$$
S\left(H_{1}, H_{2}\right)=G\left(H_{1}, H_{2}\right)-G^{1}\left(H_{1}\right)-G^{2}\left(H_{2}\right) .
$$

It follows that:

$$
\frac{\partial^{2} S}{\partial H_{1} \partial H_{2}}\left(H_{1}, H_{2}\right)=\frac{\partial^{2} G}{\partial H_{1} \partial H_{2}}\left(H_{1}, H_{2}\right)=\alpha(1+\alpha) \tau K W\left(H_{1}+\tau H_{2}\right)^{\alpha-1}>0
$$

We conclude that the function $S$ is supermodular in the spouses' human capital. In particular, in the absence of the non-monetary component of individual utilities, matching should be strictly positive assortative on human capital.

Lastly, equilibrium conditions determine the surplus allocation between spouses. Specifically, let $U\left(H_{1}\right)$ and $V\left(H_{2}\right)$ respectively denote, in a couple $\left(H_{1}, H_{2}\right)$, the share of surplus received by spouse 1 and 2. Feasibility implies that:

$$
U\left(H_{1}\right)+V\left(H_{2}\right)=S\left(H_{1}, H_{2}\right)
$$

while stability requires that for all $\left(H_{1}^{\prime}, H_{2}^{\prime}\right)$ :

$$
U\left(H_{1}\right)+V\left(H_{2}\right) \geq S\left(H_{1}^{\prime}, H_{2}^{\prime}\right) .
$$

It follows that

$$
U\left(H_{1}\right)=\max _{H_{2}}\left(S\left(H_{1}, H_{2}\right)-V\left(H_{2}\right)\right)
$$

and by the envelope theorem:

$$
U^{\prime}\left(H_{1}\right)=\frac{\partial S}{\partial H_{1}}\left(H_{1}, H_{2}\right) .
$$

This expression becomes

$$
U^{\prime}\left(H_{1}\right)=(\alpha+1) K W\left(H_{1}+\tau H_{2}\right)^{\alpha}
$$

and similarly

$$
V^{\prime}\left(H_{2}\right)=\tau(\alpha+1) K W\left(H_{1}+\tau H_{2}\right)^{\alpha} .
$$

These derivatives are important because they represent the additional gain generated, in the marriage market, by a marginal increase in the stock of human capital. As such, they determine individuals' incentives to invest in their own human capital.

We see that

$$
\frac{U^{\prime}\left(H_{1}\right)}{V^{\prime}\left(H_{2}\right)}=\frac{1}{\tau}>1 .
$$

In other words, while both spouses gain ifrom investing in education (both derivatives are positive), the marginal gain is higher for the more educated spouse (i.e., $U^{\prime}\left(H_{1}\right)>V^{\prime}\left(H_{2}\right)$ ). This conclusion is a direct consequence of the specialization pattern, whereby the low-wage spouse is in charge of chores: 
the benefits of a larger stock of human capital do not extend to time spent on chores. In that sense, the equilibrium is, as argued above, "self-reinforcing": in a world in which one gender is more educated, the equilibrium logic leads to specialization on chores, which in turn results in stronger incentives to invest for the educated gender - thus reinforcing the initial asymmetry. Equilibrium phenomena tend therefore to exacerbate initial differences, typically resulting in strong responses to even minor changes in the economic context. Note, however, that the discrepancy decreases when time devoted to chores is reduced (i.e., in our model, when $\tau$ increases); and also that the identity of the lower-wage spouse depends on $H_{1} \gtrless H_{2}$.

Discrete version In the empirical application below, individual human capital will be proxied by the person's education. Each individual $i$ belongs to an education class $I=1, \ldots, \mathcal{K}$ which is observed by the econometrician. Let $S^{I J}$ denote the economic surplus generated by the matching of spouses with respective educations $I$ and $J$.

To measure supermodularity with discrete types, cross derivatives must be replaced with cross differences. We call a $2 \times 2$ matrix $\left(\begin{array}{cc}a & b \\ c & d\end{array}\right)$ supermodular if $a+d-b-c \geq 0$. Then the $\mathcal{K} \times \mathcal{K}$ matrix $S$ is supermodular on the diagonal iff all its $2 \times 2$ diagonal submatrices are supermodular.

Equivalently, define the cross-difference operator $D^{I J, K L}$ by:

$$
D^{I J, K L}(A)=A^{I J}+A^{K L}-A^{I L}-A^{K J}
$$

for any matrix $A$. In particular, let the supermodular core of the surplus matrix $S$ be the set of numbers

$$
\mathcal{D}(S)=\left(D^{I I, K K}(S), I, K=1, \ldots, \mathcal{K}, I<K\right)
$$

Then the surplus is supermodular on the diagonal if and only if all components of its supermodular core are positive (this is very similar to the definition in Siow 2015.)

\subsubsection{Comparative statics: general results}

Driving forces The theoretical model presented above generates clear-cut comparative statics results. As argued in the introduction, we concentrate on two major changes that affected household technology over the recent decades. One is the sustained rate of technological advance in the household sector-what Greenwood et al. (2005) called "engines of liberation". Although the process started early (around the beginning of the twentieth century, for instance, for refrigerators), progress continued regularly since then. The latest wave of technological innovations includes dishwashers (whose current penetration rate is around $80 \%$ in the US, against $45 \%$ in 1990 and less than $10 \%$ in the sixties) and microwaves (which appeared in the $80 \mathrm{~s}$, for a current penetration rate over $95 \%$ ). Greenwood et al. convincingly argue that "technological progress in the 
household sector played a major role in liberating women from the home" (p. 109). In our model, the direct translation is a significant reduction of the time $\tau^{\prime}$ devoted to chores - or, equivalently, an increase in available time $\tau$.

A second and major evolution is the increased importance of human capital for wages and income. This trend is well summarized by Goldin and Katz (2009, p. 2):

[...] much of the rising wage inequality in recent history can be traced to rising differences between the wages of the highly educated and the less educated ${ }^{17}$.

In other words, while a child's future (expected) well-being has always been an increasing function of the accumulated stock of human capital, this relationship is stronger and steeper now than it was fifty years ago. In our model, the direct implication of this trend is that the coefficient $\alpha$, which summarizes the impact of human capital on future income and well-being, has increased significantly over the period under consideration.

Note, however, that the increased importance of human capital has a double effect. Not only does it boost the returns on investment on children, but it also directly affects the link between the parents' stock of human capital and the wage they receive on the market. Again, the theoretical framework delivers precise predictions regarding this impact. The parameter governing the relationship between wages and human capital is the aggregate wage shock $W$; in our comparative statics analysis, we shall therefore investigate the consequences of an increase in $W$, particularly for more educated people.

Lastly, the higher level of female human capital implies that the opportunity cost of the time women devote to non market activities - both chores and children - is much higher. Moreover, wives have become more likely to earn more than their husbands. While the fraction of couples where husband and wife have the same education has remained more or less constant, couples with a more educated wife now outnumber those exhibiting the opposite pattern; and in more than $20 \%$ of couples, the wife's income exceeds the huband's. Note that these trends are by no means specific to the US; they affect all developed economies, and many developing ones as well.

Implied predictions: domestic time use Given our structural model, these evolutions should generate two types of testable consequences. A first set of predictions can directly be tested on available data. According to our model, the total time devoted to chores should decrease. Moreover, and given our assumption that male and female time are perfect substitutes, the specialization patterns should shift, with fewer women and more men devoting time to such domestic activities.

\footnotetext{
${ }^{17}$ Goldin and Katz estimate that increases in the economic returns to investments in education from 1973 to 2005 account for about 60 percent of the rise in wage inequality over that period. For a detailed analysis, see Goldin and Katz (2008)
} 
Next, consider investment in children in a couple in which the husband earns higher wages. A first prediction is that the time spent by the husband should increase. Indeed, efficiency implies that:

$$
t_{1}=\frac{\alpha \beta}{1+\alpha}\left(1+\tau \frac{H_{2}}{H_{1}}\right) \text {. }
$$

The right-hand side is increasing in $\alpha, \tau$, and in the ratio $H_{2} / H_{1}$-all of which have increased over the period - and is independent of the wage coefficient $W$. Things are more complex for women as (2) gives

$$
t_{2}=\frac{\alpha(1-\beta)}{1+\alpha}\left(\frac{H_{1}}{H_{2}}+\tau\right) .
$$

Here, $t_{2}$ is increasing in $\alpha$ and $\tau$ and does not depend on $W$; but it decreases with the ratio $H_{2} / H_{1}$. Therefore, the time the wife spends with children will increase only if the first two effects - increased importance of these investments and more time available - dominate the third, i.e. the shift in the education profile within couples. All in all, we expect the increase (if any) to be smaller than for men.

Implied predictions: supermodularity and its evolution A second set of predictions relates to the evolution of matching patterns. Our model generates a supermodular surplus, and it is natural to ask whether this supermodularity is strengthened or weakened by the various trends we just described. To answer this question, let us first see how the second cross derivative - whose sign determines super- or submodularity - changes with the key parameters. (3) gives:

$$
\frac{\partial^{3} S}{\partial \tau \partial H_{1} \partial H_{2}}\left(H_{1}, H_{2}\right)=\frac{\alpha^{\alpha+1} \beta^{\alpha \beta} W\left(H_{1}+\tau H_{2}\right)^{\alpha-2}\left(H_{1}+\alpha \tau H_{2}\right)}{(\alpha+1)^{\alpha}(1-\beta)^{\alpha(\beta-1)}}>0
$$

and

$\frac{\partial^{3} S}{\partial \alpha \partial H_{1} \partial H_{2}}\left(H_{1}, H_{2}\right)=\frac{\beta^{\alpha \beta} \alpha^{\alpha+1} \tau W\left(H_{1}+\tau H_{2}\right)^{\alpha-1}}{(1-\beta)^{\alpha(\beta-1)}(\alpha+1)^{\alpha}}\left(\ln \left(H_{1}+\tau H_{2}\right)+L(\alpha, \beta)\right)$

where

$$
L(\alpha, \beta)=\frac{2 \alpha+1}{\alpha(\alpha+1)}+\ln \frac{\alpha}{\alpha+1}+\ln \left((1-\beta)^{1-\beta} \beta^{\beta}\right)
$$

It follows that an increase in $\tau$ always makes the surplus function $S$ more supermodular. So does an increase in $\alpha$, since the expression $L(\alpha, \beta)$ is always positive for $\alpha, \beta \in[0,1]$. Moreover, these impacts increase with both levels of human capital. Finally,

$$
\frac{\partial^{3} S}{\partial W \partial H_{1} \partial H_{2}}\left(H_{1}, H_{2}\right)=\alpha(1+\alpha) \tau K\left(H_{1}+\tau H_{2}\right)^{\alpha-1}>0
$$

so that an increase in the market reward to human capital (as summarized by the factor $W$ ) also increases the supermodularity of the surplus. 
Differences by human capital An implicit assumption of this comparative statics exercise is that the importance of human capital (either for children, as summarized by the coefficient $\alpha$, or for parental wages, as indicated by the wage coefficient $W$ ) has increased uniformly for all educational levels. In practice, the impact is mostly visible at the top of the distribution. The college and college-plus premiums have drastically increased since the 1980s. According to David Autor (2014), the earnings gap between the median high-school educated and college-educated US males working full-year, full-time jobs doubled between 1979 and $2012^{18}$. On the contrary, the return to education at a lower level (say, between high-school drop-out and high-school graduate) have probably increased much less, if at all. While an exact quantification is plagued with selection issues, Lawrence Mishel et al. (2013) estimate that the "high school premium" remained fairly stable (between 20 and $25 \%$ ) over the same period. Similarly, raw data indicate (again without controlling for selection) that the wage difference between high school graduates and "some college" actually declined over the same period.

This suggests that investment in human capital has become even more important for higher-education couples. In our model, this would correspond to a more convex $u_{i}$ functions, and to larger increases of the wage factor $W$ for individuals with nmore education. Therefore our comparative statics conclusions should apply with more force at the top than at the bottom of the distribution.

The discrete setting Our first set of predictions, which regard intra-household allocation of domestic time, are readily tested. On the other hand, predictions regarding assortative matching must be translated into our discrete setting. The crucial tool is the supermodular core matrix defined in (4) and (5). In particular, we submit that the somewhat vague notion that "assortative matching creates more surplus now than it used to in the past" should be formally defined as follows. Suppose that we observe the surplus matrix $S$ over two periods, $T=1$ and $T=2$; in particular, we can compute the supermodular core $\mathcal{D}(S)$ at each period. Then

\section{Definition 1 (Additional surplus generated by assortativeness)}

The surplus generated by assortativeness does not change between $T=1$ and $T=2$ if and only if the array $\mathcal{D}(S)$ is the same at $T=1$ and $T=2$.

The surplus generated by assortativeness increases if and only if all components of the array $\mathcal{D}(S)$ increase between $T=1$ and $T=2$; equivalently, the matrix $\left(S_{2}-S_{1}\right)$ is supermodular.

Note that this definition is of independent interest: it provides an explicit characterization of the somewhat hazy notion of "increased preferences for assortativeness" - which, in our context, corresponds to changes in the additional surplus generated by assortativeness. Moreover, this characterization is

\footnotetext{
${ }^{18}$ From 17,000 to 34,000 constant 2012 dollars.
} 
structural: it relies on a model in which assortativeness naturally relates to diagonal supermodularity of the surplus function, and it exploits this relationship to provide a formal definition.

Also note that the definition can be restricted to a subset of the categories. For instance, we shall say that the additional surplus generated by assortativeness has increased for more educated people (say, individuals at education level $\mathcal{L}$ and above $)$ if the corresponding $(\mathcal{K}-\mathcal{L}+1) \times(\mathcal{K}-\mathcal{L}+1)$ submatrix of $S$ exhibits the property in Definition 1. More precisely, define the matrix $S_{c}^{\mathcal{L}}$ by

$$
S_{c}^{\mathcal{L}}=\left(S_{c}^{I J}\right)_{I, J=\mathcal{L}, \ldots, \mathcal{K}}
$$

then we require that the matrix $S_{c^{\prime}}^{\mathcal{L}}-S_{c}^{\mathcal{L}}$ be supermodular on the diagonal for $c^{\prime}>c$. Equivalently, we require all components of the matrix

$$
\mathcal{D}\left(S_{c}^{\mathcal{L}}\right)=\left(D^{I I, K K}\left(S c^{\mathcal{L}}\right), I, K=\mathcal{L}, \ldots, \mathcal{K}, I<K\right)
$$

to increase with $c$.

With this definition, the previous comparative statics results predict that the additional surplus generated by assortativeness must have increased over the period, particularly for higher levels of education. In practice, if we estimate the economic surplus matrix $S_{c}^{\mathcal{L}}$ over two periods, $c$ and $c^{\prime}$, with $c<c^{\prime}$, the array $\mathcal{D}\left(S_{c}^{\mathcal{L}}\right)$ should increase with $c$ for $\mathcal{L}$ "large enough".

\subsection{Preferences: non monetary component}

In addition to preferences over commodities, each individual has non-monetary marital preferences which we model by random vectors. Following Choo and Siow (2006), we assume that preferences over potential spouses are individualspecific and only depend on the spouse's education class. For instance, a woman $j$ belonging to class $J$ has a vector of marital preferences

$$
\boldsymbol{b}_{j}^{J}=\left(b_{j}^{\emptyset J}, b_{j}^{1 J}, \ldots, b_{j}^{\mathcal{K} J}\right)
$$

where $b_{j}^{K J}$ denotes the utility $j$ derives from marrying a spouse with an education $K$ (and where, by convention, $b_{j}^{\emptyset J}$ denotes the utility $j$ derives from staying single). Similarly, man $i$ 's idiosyncratic marital preferences are described by the vector

$$
\boldsymbol{a}_{i}^{I}=\left(a_{i}^{I \emptyset}, a_{i}^{I 1}, \ldots, a_{i}^{I \mathcal{K}}\right)
$$

where $I$ denotes $i$ 's education. Note that the distribution of an individual's vector of marital preferences may depend on the individual's own education; more educated men may value an educated wife differently than less educated men. Not only is this assumption quite plausible empirically, but it is also needed to reflect the endogeneity of education. In CIW, for instance, individual tastes for marriage influence investment in education, because they affect the probability that an individual reaps the benefits of education on the marriage market. Since 
individuals with different marital tastes invest differently, the conditional distribution of taste given education will typically vary with education, reflecting the selection into educational choices. Consequently, we define

$$
A^{I J}=E\left(a_{i}^{I J} \mid i \in I\right) \text { and } B^{I J}=E\left(b_{j}^{I J} \mid j \in J\right) .
$$

It should be stressed thatin this framework, these idiosyncratic, additively separable shocks are the only source of unobserved heterogeneity. This assumption is crucial in order to apply the Choo-Siow approach; see Chiappori and Salanié (2016) for a detailed discussion.

Finally, and still following Choo and Siow (2006), we assume that economic and marital preferences are additively separable. To be more precise, the marital surplus $s_{i j}$ generated by the match of man $i$ with education $I$ and woman $j$ with education $J$ is the sum of two components. One is the expected economic surplus $S^{I J}$ generated by joint consumption; the other consists of the sum of the spouses' idiosyncratic preferences for marriage with each other, relative to singlehood.

$$
s_{i j}=S^{I J}+\left(a_{i}^{I J}-a_{i}^{I \emptyset}\right)+\left(b_{j}^{I J}-b_{i}^{\emptyset J}\right) .
$$

or, using the previous definitions:

$$
\begin{aligned}
s_{i j} & =\left(S^{I J}+\left(A^{I J}-A^{I \emptyset}\right)+\left(B^{I J}-B^{\emptyset J}\right)\right) \\
& +\left(\left(a_{i}^{I J}-A^{I J}\right)-\left(a_{i}^{I \emptyset}-A^{I \emptyset}\right)\right) \\
& +\left(\left(b_{j}^{I J}-B^{I J}\right)-\left(b_{j}^{\emptyset J}-B^{\emptyset J}\right)\right) .
\end{aligned}
$$

The component on the first line

$$
Z^{I J}=S^{I J}+\left(A^{I J}-A^{I \emptyset}\right)+\left(B^{I J}-B^{\emptyset J}\right)
$$

is the conditional expectation of the total surplus for matches between classes $I$ and $J$. Within it, $S^{I J}$ is the conditional expected economic surplus, while $\left(A^{I J}-A^{I \emptyset}\right)+\left(B^{I J}-B^{\emptyset J}\right)$ represents the conditional expected surplus from marital preferences. By definition, the components on the last two lines have zero expectation across all hypothetical matches. We use

$$
\alpha_{i}^{I J}=a_{i}^{I J}-A^{I J} \text { and } \beta_{j}^{I J}=b_{j}^{I J}-B^{I J}
$$

to denote the within-class variation of marital preferences; note that, by construction,

$$
E\left[\alpha_{i}^{I J}\right]=E\left[\beta_{j}^{I J}\right]=0 \text { for all } i, j, I, J
$$

Finally, the total surplus generated by the match between $i \in I$ and $j \in J$ is:

$$
s_{i j}=Z^{I J}+\left(\alpha_{i}^{I J}-\alpha_{i}^{I \emptyset}\right)+\left(b_{j}^{I J}-b_{j}^{\emptyset J}\right)
$$

The matrix $Z=\left(Z^{I J}\right)$ will play a crucial role in what follows. As we shall see, the equilibrium matching will depend on preferences through the matrix $Z$ 
and the distribution of the $\alpha$ 's and $\beta$ 's. From the definitions above, $Z^{I J}$ reflects the distribution of income and preferences over commodities of spouses who chose education levels $I$ and $J$ (and each other), as well as the distribution of their marital preferences. It is therefore a complex object; but it is the crucial construct that determines marital patterns in our context. Our goal is to find reasonable conditions under which it is identifiable from observed matching patterns.

\subsection{Matching}

A matching consists of

(i) a measure $d \mu$ on the set $\mathcal{M} \times \mathcal{F}$, such that the marginal of $d \mu$ over $\mathcal{M}$ (resp. $\mathcal{F})$ is $d \mu_{\mathcal{M}}\left(d \mu_{\mathcal{F}}\right)$; and

(ii) a set of payoffs (or imputations) $\left\{u_{i}, i \in \mathcal{M}\right\}$ and $\left\{v_{j}, j \in \mathcal{F}\right\}$ such that

$$
u_{i}+v_{j}=z_{i j} \text { for any }(i, j) \in \operatorname{Supp}(d \mu)
$$

In words, a matching indicates who marries whom (note that the allocation may be random, hence the measure), and how each married couple shares the surplus $z_{i j}$ generated by their match. The numbers $u_{i}$ and $v_{j}$ are the expected utilities man $i$ and woman $j$ get on the marriage market, on top of their utilities when they remain single;. In any pair $(i, j)$ that marries with positive probability, $u_{i}$ and $v_{j}$ must add up to the total surplus generated by the union.

\subsubsection{Stable Matchings}

A matching is stable if one can find neither a man $i$ who is currently married but would rather be single, nor a woman $j$ who is currently married but would rather be single, nor a woman $j$ and a man $i$ who are not currently married together but would both rather be married together than remain in their current situation. Formally, we must have that:

$$
\begin{aligned}
u_{i} & \geq 0 \text { for any } i \in \mathcal{M} \\
v_{j} & \geq 0 \text { for any } j \in \mathcal{F} \\
u_{i}+v_{j} & \geq z_{i j} \text { for any }(i, j) \in \mathcal{M} \times \mathcal{F} .
\end{aligned}
$$

The two conditions in (10) and (11) implies that married agents would not prefer remaining single. Condition (12) states that for any possible match $(i, j), i$ and $j$ could not both strictly increase their utility by matching together.

As is well known, stable matchings maximize the total joint surplus ${ }^{19}$. Specifically, consider the set $\mathcal{D}_{\mathcal{M} \times \mathcal{F}}$ of measures $d \mu$ over the product space

\footnotetext{
${ }^{19}$ See for instance Chiappori, McCann and Nesheim (2010) or Chiappori (2017) for a detailed exposition.
} 
$\mathcal{M} \times \mathcal{F}$ whose marginal over $\mathcal{M}$ (resp. $\mathcal{F}$ ) is $d \mu_{\mathcal{M}}$ (resp. $d \mu_{\mathcal{F}}$ ); and within this set, find the measure that maximizes total surplus:

$$
\Sigma=\max _{\mu \in \mathcal{D}_{\mathcal{M} \times \mathcal{F}}} \int z d \mu
$$

This is an optimal transportation problem, which is linear in its unknown (i.e. the measure $\mu$ ). It therefore admits a dual, which consists in finding $\left\{u_{i}, i \in \mathcal{M}\right\}$ and $\left\{v_{j}, j \in \mathcal{F}\right\}$ that minimize

$$
\int u_{i} d \mu_{\mathcal{M}}(i)+\int v_{j} d \mu_{\mathcal{F}}(j)
$$

under the constraints (10), (11) and (12).

One can show that if a matching is stable, the corresponding measure $d \mu$ solves the surplus maximization problem. Conversely, if the surplus maximization problem has a solution $d \mu$, then the dual problem has solutions $\left\{u_{i}, i \in \mathcal{M}\right\}$ and $\left\{v_{j}, j \in \mathcal{F}\right\}$, and the matching defined by $d \mu,\left\{u_{i}, i \in \mathcal{M}\right\}$ and $\left\{v_{j}, j \in \mathcal{F}\right\}$ is stable.

A first consequence is that existence of a stable matching is guaranteed under mild continuity assumptions: we simply need the (linear) surplus maximization problem to have a solution. Moreover, the dual of this maximization problem generates, for each man $i$ (resp. woman $j$ ), a dual variable or "shadow price" $u_{i}$ (resp. $v_{j}$ ); and these dual variables exactly coincide with payoffs associated to the matching problem. Finally, note that with finite populations, the payoffs $u_{i}$ and $v_{j}$ are not uniquely defined: they can be marginally altered without violating the finite set of inequalities (10)-(12). However, when the populations become large, the intervals within which $u_{i}$ and $v_{j}$ may vary typically shrink; in the limit of continuous and atomless populations, the distributions of the individual payoffs are exactly determined.

\subsubsection{A basic lemma}

From an economic perspective, our main interest lies in the dual variables $u$ and $v$. Indeed, $v_{j}$ is the additional utility provided to woman $j$ by her equilibrium marriage outcome. This value is individual-specific, as it depends on Ms $j$ 's preferences for marriage; but its expected value conditional on $j$ having reached a given level of education $J$ is directly related to the marital premium associated with education $J$ (more on this below).

In our context, there exists a simple and powerful characterization of these dual variables:

Lemma 2 For any stable matching, there exist numbers $U^{I J}$ and $V^{I J}$, defined for $I, J=1, \ldots, \mathcal{K}$, with

$$
U^{I J}+V^{I J}=Z^{I J}
$$


satisfying the following property: for any matched couple $(i, j)$ such that $i \in I$ and $j \in J$,

$$
\begin{aligned}
u_{i}= & U^{I J}+\left(\alpha_{i}^{I J}-\alpha_{i}^{0 J}\right) \\
& \text { and } \\
v_{j}= & V^{I J}+\left(\beta_{j}^{I J}-\beta_{j}^{0 J}\right)
\end{aligned}
$$

Proof. Assume that $i$ and $i^{\prime}$ both belong to $I$, and their partners $j$ and $j^{\prime}$ both belong to $J$. Stability requires that:

$$
\begin{aligned}
u_{i}+v_{j} & =Z^{I J}+\left(\alpha_{i}^{I J}-\alpha_{i}^{0 J}\right)+\left(\beta_{j}^{I J}-\beta_{j}^{0 J}\right) \\
u_{i}+v_{j^{\prime}} & \geq Z^{I J}+\left(\alpha_{i}^{I J}-\alpha_{i}^{0 J}\right)+\left(\beta_{j^{\prime}}^{I J}-\beta_{j^{\prime}}^{0 J}\right) \\
u_{i^{\prime}}+v_{j^{\prime}} & =Z^{I J}+\left(\alpha_{i^{\prime}}^{I J}-\alpha_{i^{\prime}}^{0 J}\right)+\left(\beta_{j}^{I J}-\beta_{j}^{0 J}\right) \\
u_{i^{\prime}}+v_{j} & \geq Z^{I J}+\left(\alpha_{i^{\prime}}^{I J}-\alpha_{i^{\prime}}^{0 J}\right)+\left(\beta_{j^{\prime}}^{I J}-\beta_{j^{\prime}}^{0 J}\right)
\end{aligned}
$$

Subtracting (15) from (16) and (18) from (17) gives

$$
\left(\beta_{j^{\prime}}^{I J}-\beta_{j^{\prime}}^{0 J}\right)-\left(\beta_{j}^{I J}-\beta_{j}^{0 J}\right) \leq v_{j^{\prime}}-v_{j} \leq\left(\beta_{j^{\prime}}^{I J}-\beta_{j^{\prime}}^{0 J}\right)-\left(\beta_{j}^{I J}-\beta_{j}^{0 J}\right)
$$

hence

$$
v_{j^{\prime}}-v_{j}=\left(\beta_{j^{\prime}}^{I J}-\beta_{j^{\prime}}^{0 J}\right)-\left(\beta_{j}^{I J}-\beta_{j}^{0 J}\right)
$$

It follows that the difference $v_{j}-\left(\beta_{j}^{I J}-\beta_{j}^{0 J}\right)$ does not depend on $j$, i.e.:

$$
v_{j}-\left(\beta_{j}^{I J}-\beta_{j}^{0 J}\right)=V^{I J} \quad \text { for all } i \in I, j \in J
$$

The proof for $u_{i}$ is identical.

In words, Lemma 2 states that the dual utility $v_{j}$ of woman $j$, belonging to class $J$ and married with a husband in education class $I$, is the sum of two terms. The first one is woman $j$ 's idiosyncratic preference for a spouse with education $I$ over singlehood, $\beta_{j}^{I J}-\beta_{j}^{\emptyset J}$; the second term, $V^{I J}$, only depends on the spouses' classes, not on who they are. In terms of surplus division, therefore, the $U^{I J}$ and $V^{I J}$ denote how the deterministic component of the surplus, $Z^{I J}$, is divided between spouses; then a spouse's utility is the sum of this share of the common component and of his or her idiosyncratic contribution.

Finally, for notational consistency, we define $U^{I \emptyset}=V^{\emptyset J}=0$ for all $I$ and $J$.

\subsubsection{Stable matching: a characterization}

An immediate consequence of Lemma 2 is that the stable matching has a simple characterization in terms of individual choices:

Proposition 3 A set of necessary and sufficient conditions for stability is that 
1. for any matched couple $(i \in I, j \in J)$ one has

$$
\alpha_{i}^{I J}-\alpha_{i}^{I K} \geq U^{I K}-U^{I J} \text { for all } K=\emptyset, 1, \ldots, \mathcal{K}
$$

and

$$
\beta_{j}^{I J}-\beta_{j}^{K J} \geq V^{K J}-V^{I J} \text { for all } K=\emptyset, 1, \ldots, \mathcal{K}
$$

2. for any single man $i \in I$ one has

$$
\alpha_{i}^{I J}-\alpha_{i}^{I \emptyset} \leq-U^{I J} \text { for all } J
$$

3. for any single woman $j \in J$ one has

$$
\beta_{j}^{I J}-\beta_{j}^{\emptyset J} \leq-V^{I J} \text { for all } I
$$

Proof. See Appendix A.

Stability thus readily translates into a set of inequalities in our framework; and each of these inequalities relates to one agent only. This property is crucial, because it implies that the model can be estimated using standard statistical procedures applied at the individual level, without considering conditions on couples. This separation is possible because the endogenous factors $U^{I J}$ and $V^{I J}$ adjust to make the separate individual choices consistent with each other.

\subsection{Interpretation and comparative statics}

\subsubsection{The marital college premium}

Labor economists define the "college premium" as the percentage increase in the wage rate that can be expected from a college education. This wage premium can readily be measured using available data, after controlling for selection into college; existing work suggests that it is similar for singles and married persons and for men and women ${ }^{20}$. We used the PSID to estimate a simple Mincer equation, separately for each gender and race but without any attempt to control for selection. Figure 13 shows the resulting estimates of the labor market college premium for both genders, for whites and for blacks. Our paper is mostly concerned with changes in the college premium over time. It is worth noting at this stage that these (admittedly coarse) estimates suggest that if anything, the labor market college premium has increased more for men than for women. Also note the striking discrepancy between whites and blacks, and in particular the decrease in the labor market value of a college degree for black women over time.

CIW point out that in addition to this wage premium, there exists a marital college premium: a college education enhances an individual's marital prospects, via the probability of being married and the expected education (or income) of the spouse, but also the size of the surplus generated and its division within

${ }^{20}$ See for instance Bronson (2014, Figure 3B). 
the couple. In other words, it is well-understood that college education benefits individuals in terms of higher wages, better career prospects, etc; but our goal here is to capture the additional benefits that college-educated individuals receive on the marriage market.

[Figure 13 about here.]

The notions previously defined allow a clear definition of the marital college premium. Indeed, the surplus is computed as the difference between the total utility generated within the couple and the sum of individual utilities of the spouses if single, thus capturing exactly the additional gains from education that only benefit married people. Regarding individual well-being, an intuitive interpretation of $U^{I J}$ (or equivalently of $V^{I J}$ ) would be the following. Assume that a man randomly picked in class $I$ is forced to marry a woman belonging to class $J$ (assuming that the populations are large, so that this small deviation from stability does not affect the equilibrium payoffs). Then his expected utility is exactly $U^{I J}$ (the expectation being taken over the random choice of the individual - therefore of his preference vector - within the class).

Note, however, that this value does not coincide with the average utility of men in class $I$ who end up being married to women $J$ in a stable matching. The latter value is larger than $U^{I J}$, reflecting the fact that agents choose their spouses. The expected surplus of an agent with education $I$ is in fact

$$
\bar{u}^{I}=E \max _{J=0,1, \ldots, \mathcal{K}}\left(U^{I J}+\alpha^{I J}\right),
$$

where the expectation is taken upon the distribution of the preference shock $\alpha$. This expected surplus depends on the distribution of the preference shocks; it will be computed below under a specific assumption on this distribution.

\section{Empirical implementation}

We now describe the econometric model we shall take to data. Galichon and Salanié (2015) proved that separable models are just identified under the strong condition that the econometrician has exact knowledge of the probability distributions of $\alpha$ and $\beta$. This is an obvious weakness, since it implies that the model is simply not testable from cross-sectional data. A crucial feature of our approach is that we analyze multiple markets; in practice, we shall consider several cohorts indexed by $c=1, \ldots, T$ and exploit the time variations in the education profiles of the populations at stake. Such a setting, in principle, should allow us to relax some restrictions while still generating overidentification tests.

Our first task is to describe how the structural components of our statistical framework evolve across cohorts. We start, as a benchmark, with an immediate generalization of CS. We show that this benchmark version generates strong overidentifying restrictions, and we describe a set of overidentification tests. This model fits the data well for the black population, but it is strongly rejected for whites. We then discuss several further extensions of the model, which we go on to test. 


\subsection{The benchmark version}

\subsubsection{The structural framework}

In a static version of CS, the surplus generated by the match of man $i$, belonging to class $I$, with woman $j$, belonging to class $J$, takes the form:

$$
z_{i j}=Z^{I J}+\left(\alpha_{i}^{I J}-\alpha_{i}^{I \emptyset}\right)+\left(\beta_{j}^{I J}-\beta_{j}^{\emptyset J}\right)
$$

In a multi-cohorts setting, it is natural to assume that the shocks are independent across cohorts. How the deterministic part $Z^{I J}$ varies with time is less clear. Allowing the entire $\boldsymbol{Z}$ matrix to vary freely across cohorts would amount to independently repeating static versions of CS, with no gain in terms of testability. Our benchmark model, therefore, introduces category-specific drifts, whereby the $Z^{I J}$ terms vary according to:

$$
Z_{c}^{I J}=\zeta_{c}^{I}+\xi_{c}^{J}+Z^{I J}
$$

so that

$$
z_{i j, c}=Z^{I J}+\zeta_{c}^{I}+\xi_{c}^{J}+\left(\alpha_{i, c}^{I J}-\alpha_{i, c}^{I \emptyset}\right)+\left(\beta_{j, c}^{I J}-\beta_{j, c}^{\emptyset J}\right)
$$

In practice, the drifts $\zeta_{c}^{I}$ and $\xi_{c}^{J}$ capture possible changes over time in the surplus generated by marriage. There are several reasons to expect the surplus to vary across periods. One is that technological innovations have drastically altered the technology of domestic production, therefore the respective gender roles within the household (see Greenwood et al 2005.) Other important factors were the evolution of fertility control, as emphasized by Michael (2000) and Goldin and Katz (2002) among others; and improvements in medical techniques and in infant feeding (Albanesi and Olivetti 2016.) Finally, remember that in our framework, the systematic part of the surplus, $Z^{I J}$, can be interpreted as a reduced form for more dynamic interactions, including divorce and remarriage; as a consequence, changes in divorce laws or remarriage probabilities may affect the surplus.

It is important to stress what the proposed extension allows and what it rules out. Under (23), the benefits of marriage may evolve over time; and these evolutions may be both gender- and education- specific. We allow, for instance, the reduction in the gains generated by marriage to be different for an educated women and for an unskilled man. ${ }^{21}$

However, all models of the form (23) satisfy an important property: the supermodular core is the same for all cohorts. To see why, simply note that

\footnotetext{
${ }^{21}$ In addition, the coefficients $\xi$ and $\zeta$ can also capture changes in the correlation between education and mean marital preferences across cohorts (e.g., single women gaining more from education over time).
} 
under (23):

$$
\begin{aligned}
D^{I J, K L}\left(Z_{c}\right) & =Z_{c}^{I J}-Z_{c}^{I L}-Z_{c}^{K J}+Z_{c}^{K L} \\
& =\left(\zeta_{c}^{I}+\xi_{c}^{J}+Z^{I J}\right)-\left(\zeta_{c}^{I}+\xi_{c}^{L}+Z^{I L}\right) \\
& -\left(\zeta_{c}^{K}+\xi_{c}^{J}+Z^{K J}\right)+\left(\zeta_{c}^{K}+\xi_{c}^{L}+Z^{K L}\right) \\
& =Z^{I J}-Z^{I L}-Z^{K J}+Z^{K L}=D^{I J, K L}(Z) .
\end{aligned}
$$

In this new setting, Lemma 2 has an immediate generalization:

\section{Corollary 4}

For any cohort $c$, there exist numbers $U_{c}^{I J}$ and $V_{c}^{I J}, I, J=1, \ldots, \mathcal{K}$, with

$$
U_{c}^{I J}+V_{c}^{I J}=Z_{c}^{I J}
$$

satisfying the following property: for any matched couple $(i, j)$ in cohort $c$ such that $i \in I$ and $j \in J$,

$$
\begin{aligned}
u_{i}= & U_{c}^{I J}+\left(\alpha_{i}^{I J}-\alpha_{i}^{I \emptyset}\right) \\
& \text { and } \\
v_{j}= & V_{c}^{I J}+\left(\beta_{j}^{I J}-\beta_{j}^{\emptyset J}\right) .
\end{aligned}
$$

\subsubsection{Distributions}

Next we need to describe the probability distributions of the random terms $\boldsymbol{\alpha}$ and $\boldsymbol{\beta}$. Having transformed the problem into a standard discrete choice problem, it is natural to make the following assumption:

Assumption 5 (Gumbel) The random terms $\alpha_{i}^{I J}$ and $\beta_{j}^{I J}$ follow independent Gumbel $^{22}$ distributions $G(-k, 1)$, with $k \simeq 0.5772$ the Euler constant.

In particular, the $\alpha_{i}^{I J}$ and $\beta_{j}^{I J}$ have mean zero and variance $\frac{\pi^{2}}{6}$. The model can easily be extended to allow for covariates; this extension is fully described in Chiappori, Salanié and Weiss (2011).

A direct consequence of Proposition 3 is that, for any $I$ and any $i \in I$ in cohort $c$ :

$$
\begin{aligned}
\gamma_{c}^{I J} & \equiv \operatorname{Pr}(i \text { matched with a woman in } J) \\
& =\frac{\exp \left(U_{c}^{I J}\right)}{\sum_{K} \exp \left(U_{c}^{I K}\right)+1}
\end{aligned}
$$

and

$$
\gamma_{c}^{I \emptyset} \equiv \operatorname{Pr}(i \text { single })=\frac{1}{\sum_{K} \exp \left(U_{c}^{I K}\right)+1}
$$

\footnotetext{
${ }^{22}$ This distribution is also referred to as the "type-I extreme value distribution." While it has been used in economics since Daniel McFadden (1973), Dagsvik (2000) and Choo and Siow (2006) were the first to apply it to the study of marriage markets.
} 
Similarly, for any $J$ and any woman $j \in J$ in cohort $c$ :

$$
\begin{aligned}
\delta_{c}^{I J} & \equiv P(j \text { matched with a man in } I) \\
& =\frac{\exp \left(V_{c}^{I J}\right)}{\sum_{K} \exp \left(V_{c}^{K J}\right)+1} \text { and } \\
\delta_{c}^{\emptyset J} & \equiv P(j \text { single })=\frac{1}{\sum_{K} \exp \left(V_{c}^{K J}\right)+1}
\end{aligned}
$$

These formulas can be inverted to give:

$$
U_{c}^{I J}=\ln \left(\frac{\gamma_{c}^{I J}}{1-\sum_{K} \gamma_{c}^{I K}}\right)
$$

and

$$
V_{c}^{I J}=\ln \left(\frac{\delta_{c}^{I J}}{1-\sum_{K} \delta_{c}^{K J}}\right) .
$$

In what follows, we assume that there are singles in each class (a claim obviously supported by the data); therefore $\gamma_{c}^{I \emptyset}>0$ and $\delta_{c}^{\emptyset J}>0$ for each $I, J$, implying that $\sum_{K} \gamma_{c}^{I K}<1$ and $\sum_{K} \delta_{c}^{K J}<1$ for all $I, J$.

We can readily compute the class-specific expected utilities

$$
\bar{u}^{I}=E\left[\max _{J=\emptyset, 1, \ldots, \mathcal{K}}\left(U_{c}^{I J}+\alpha_{i}^{I J}\right)\right]
$$

Under our assumptions, the difference $\bar{u}^{I}-\bar{u}^{K}$ denotes the difference in expected surplus obtained by reaching the education level $I$ instead of $K$. It therefore represents exactly the marital premium generated by that change in education level - that is, the gain that accrues to married people, on top of the benefits that singles also receive.

From the properties of Gumbel distributions, we have

$$
\bar{u}^{I}=E\left[\max _{J}\left(U_{c}^{I J}+\alpha_{i}^{I J}\right)\right]=\ln \left(\sum_{J} \exp \left(U_{c}^{I J}\right)+1\right)=-\ln \left(\gamma_{c}^{I \emptyset}\right)
$$

and similarly

$$
\bar{v}^{J}=\ln \left(\sum_{I} \exp \left(V_{c}^{I J}\right)+1\right)=-\ln \left(\delta_{c}^{\emptyset J}\right) .
$$

These results illustrate a well-known property of homoskedastic multinomial logit models: the expected utilities of participants are fully summarized by their probability of remaining single ${ }^{23}$. Two remarks can be made at this point. First, it is important to stress that there is no automatic relationship between changes in population composition (say, an exogenous increase in the proportion of women with a college degree) and their impact on expected utility. Even in

${ }^{23}$ As we will see, this property no longer holds in an heteroskedastic version of the model. 
this benchmark version, such an increase may either boost or deflate expected utility of educated women, depending on its actual consequences on probability of singlehood. Second, the one-to-one relationship between expected marital gain (by gender and education) and the corresponding percentage of singles is not robust to a generalization of the stochastic framework. Two possible generalizations seem of particular interest. First, as emphasized by Galichon and Salanié (2015), our theoretical approach could apply to any stochastic distribution; one simply has to compute the corresponding "generalized entropy", at the cost of an increase in the complexity of estimation. Less ambitiously, an heteroskedastic version of CS remains identifiable in our multi-market approach; we explore this idea in section 4.2.2.

Looking back at the descriptive statistics presented in section 2.2, one striking fact is the much smaller decline in marriage probability for educated women than uneducated ones. The theoretical interpretation is that, although the gain from marriage declined for all women, the decline was less pronounced for educated women; this translates into a strong increase in the marital college (and college-plus) premium, which directly reflects the difference between these gains. Moreover, this pattern is gender-specific for the white population, but not for African-Americans; we shall return to this difference.

\subsubsection{Empirical tests}

Observing several cohorts generates strong overidentifying restrictions on the benchmark model. One can actually give a simple representation of these restrictions. Start with the basic relation (23). Together with (28) and (29), this gives:

$$
\ln \left(\frac{\gamma_{c}^{I J}}{1-\sum_{H} \gamma_{c}^{I H}}\right)+\ln \left(\frac{\delta_{c}^{I J}}{1-\sum_{H} \delta_{c}^{H J}}\right)=\zeta_{c}^{I}+\xi_{c}^{J}+Z^{I J}
$$

Now remember the cross-difference operator we introduced in (4):

$$
D^{I J, K L}(A)=A^{I J}+A^{K L}-A^{I L}-A^{K J},
$$

and apply it to (32). The term $\ln \left(1-\sum_{H} \gamma_{c}^{I H}\right)$ on the left-hand side only depends on $c$ and $I$, and taking it through the cross-difference operator gives zero. Similarly, the term $\ln \left(1-\sum_{H} \gamma_{c}^{H J}\right)$ also contributes zero, as do the terms $\zeta_{c}^{I}$ and $\xi_{c}^{J}$ on the right-hand side. We are left with

$$
D^{I J, K L}\left(\ln \gamma_{c}+\ln \delta_{c}\right)=D^{I J, K L} Z,
$$

denoting $\gamma_{c}, \delta_{c}$ and $Z$ the matrices that collect respectively the $\gamma_{c}^{I J}, \delta_{c}^{I J}$ and $Z^{I J}$ terms.

But since the right-hand side does not depend on the cohort index $c$, it follows that the expression

$$
d_{c}^{I J, K L}=D^{I J, K L}\left(\ln \gamma_{c}+\ln \delta_{c}\right)
$$

should not depend on $c$. 
Two remarks can be made at this point. First, these conditions are necessary and sufficient; if they are satisfied, then one can always find some matrices $U, V$ and $Z$ and some vectors $\zeta$ and $\xi$ such that all previous conditions are satisfied. Second, they are quite restrictive. It is easy to see that with 5 classes, there are only $4 \times 4=16$ a priori independent cross-differences (see Fox, Hsu and Yang 2015.) Take the corresponding $16 T$-dimensional vectors $d^{I J K L}=$ $\left(d_{c}^{I J, K L}, c=1, \ldots, T\right)$; our assumption (23) amounts to requiring that each of them be constant (proportional to $(1, \ldots, 1)$.) This generates $16 \times(T-1)$ restrictions, a large number since we use several decades of yearly data.

\subsection{Extensions}

We shall now consider three extensions of the model that we will take to the data.

\subsubsection{Linear and Quadratic Supermodularity Trends}

Our benchmark model posits that preferences for assortativeness do not change over the period. As we shall see, this hypothesis is not rejected for the AfricanAmerican sample; but it is excessively restrictive for the white population. A natural extension is to allow preferences for assortativeness to change over the period. This should be done parsimoniously, since we want to preserve the strong testability of the model. We first introduce linear time trends in the supermodular core of the deterministic matrix. To do this, we assume that:

$$
Z_{c}^{I J}=\zeta_{c}^{I}+\xi_{c}^{J}+a^{I J}+b^{I J} \times c .
$$

Then (32) implies that

$$
d_{c}^{I J, K L}=D^{I J, K L}\left(\ln \gamma_{c}+\ln \delta_{c}\right)
$$

is a linear function of the cohort index $c$.

Given the choice of the reference categories, the number of parameters is increased by $4 \times 4=16$, and the model remains testable: the 16 a priori independent $T$-dimensional vectors $d^{I J, K L}=\left(d_{c}^{I J, K L}, c=1, \ldots, T\right)$ must now all

belong in the space spanned by the two vectors $(1, \ldots, 1)$ and $(1, \ldots, T)$. The resulting $16 \times(T-2)$ conditions are again necessary and sufficient. Moreover,

$$
D^{I J, K L}\left(Z_{c}\right)=Z^{I J}-Z^{I L}-Z^{K J}+Z^{K L}+\left(b^{I J}-b^{I L}-b^{K J}+b^{K L}\right) c
$$

and it follows from Definition 1 that the surplus generated by assortativeness increases if and only if the matrix $B=\left(b^{I J}\right)$ is supermodular on the diagonal, that is if and only if

$$
b^{I I}-b^{I K}-b^{K I}+b^{K K}>0
$$

for all $I<K$. In what follows, we therefore pay a particular attention to the supermodularity of $B$, and especially to the submatrix corresponding to higher education levels. 
Going one step further, one can introduce a quadratic trend in the time variation of the deterministic component of the surplus. Then the vectors $d^{I J, K L}$ must lie in the space spanned by the three vectors $(1, \ldots, 1),(1, \ldots, T)$ and $\left(1, \ldots, T^{2}\right)$, for a total of $16 \times(T-3)$ testable predictions.

\subsubsection{Heteroskedasticity}

A second generalization introduces class-dependent heteroskedasticity. Specifically, we consider a stochastic formulation of the type:

$$
z_{i j, c}=Z^{I J}+\sigma^{I} \alpha_{i, c}^{I J}+\mu^{J} \beta_{j, c}^{I J}
$$

where the variance of the stochastic component for a man belonging to education class $I$ depends on the parameter $\sigma^{I}$, and the variance of the stochastic component for a woman belonging to education class $J$ is determined by the parameter $\mu^{J}$. Note that we need to normalize one of these parameters.

The previous relationships can be extended to this more general setting; the reader is referred to Chiappori, Salanié and Weiss (2011). In particular, the expected surplus of a male agent in education class $I$ now is

$$
\bar{u}^{I}=\sigma^{I} \ln \left(\sum_{J} \exp \left(U^{I J} / \sigma^{I}\right)+1\right)=-\sigma^{I} \ln \left(\gamma_{c}^{I 0}\right)
$$

so that the marital education premium between classes $I$ and $K$ (representing the gain in expected surplus from belonging to $I$ instead of $K$ ) becomes:

$$
\bar{u}^{I}-\bar{u}^{K}=\sigma^{K} \ln \left(\gamma_{c}^{K 0}\right)-\sigma^{I} \ln \left(\gamma_{c}^{I 0}\right)
$$

An important result, proved in Chiappori, Salanié and Weiss (2011), is that this model is again (over) identified.

In practice, we will test whether the introduction of heteroskedasticity (resulting in $2 \times 5-1=9$ additional parameters) significantly improves the fit. ${ }^{24}$

\subsubsection{Age differences}

Finally, we may want to consider the age difference within the couple as a choice variable. The natural way to proceed is to assume that the deterministic component of the surplus, $Z$, also depends on the age difference between husband and wife, which we denote $d$. Our benchmark model, which allows for category-specific drifts, therefore becomes:

$$
Z_{c, d}^{I J}=\zeta_{c, d}^{I}+\xi_{c, d}^{J}+Z^{I J}
$$

\footnotetext{
${ }^{24} \mathrm{~A}$ model with both linear trends in supermodularity and heteroskedastic random preferences is in principle identifiable. Empirically, however, it appears to raise serious robustness issues (at least for the data under consideration), reflecting a possible overparametrization. Given our focus on the evolution of supermodularity across cohorts, we chose to concentrate on the first aspect and ignore the second.
} 
so that

$$
z_{c, d}^{I J}=Z^{I J}+\zeta_{c, d}^{I}+\xi_{c, d}^{J}+\left(\alpha_{i, c, d}^{I J}-\alpha_{i, c, d}^{I \emptyset}\right)+\left(\beta_{j, c, d}^{I J}-\beta_{j, c, d}^{\emptyset J}\right)
$$

Obviously, this model differs a lot from the initial one in both the number of observations and the number of parameters: the set of marriages of a man with education $I$, born in cohort $c$, to a woman with education $J$ is now divided into seven subcategories, each representing a specific age difference $(-4$ or less, -3 or $-2,-1$ to 1,2 or 3,4 or 5,6 or more.) In order to compare the results of this specification with those of the initial version, we concentrate on the same margins we were trying to fit initially. That is, we take a weighted average of the errors within each cell $(I, J, c)$ over the age differences $d$, and we use this averaged error to run our tests.

\section{$5 \quad$ Estimation and tests}

In order to estimate the constituent parts of our various models and to test the corresponding hypotheses, we use the minimum distance framework.

\subsection{The Benchmark Model}

Start from our benchmark model of section 4.1, with constant supermodular core and homoskedasticity. It is characterized by equation (32), which can be rewritten as

$$
\log (\operatorname{Pr}(J \mid I, c) \operatorname{Pr}(I \mid J, c))=Z_{c}^{I J} \equiv A_{I J}+B_{c}^{I}+C_{c}^{J} .
$$

This is an algebraic identity, which bears on unknown parameters. In statistical terms, it is a mixed hypothesis that constrains the unknown parameters $\left(Z_{c}^{I J}\right)$ :

$$
\exists\left(A_{I J}, B_{c}^{I}, C_{c}^{J}\right)_{I J c} \text { s.t. } \forall(I, J, c), Z_{c}^{I J}=A_{I J}+B_{c}^{I}+C_{c}^{J} .
$$

it becomes a statistical model once we take into account the sampling variation that comes from our estimates of the probabilities on the left-hand side. We obtain an estimate of the deterministic part of the joint surplus $Z_{c}^{I J}$ by combining estimates of the conditional matching probabilities:

$$
\hat{Z}_{c}^{I J}=\log (\widehat{\operatorname{Pr}}(J \mid I, c) \widehat{\operatorname{Pr}}(I \mid J, c)) .
$$

The estimated probabilities are simple averages; for instance, if there are $N_{I c}^{m}$ men of education $I$ in cohort $c$, the estimator of the probability that one of them marries a woman of education $J$ is simply the proportion of that group of men that married a women of that education.

Let $\Omega$ be the asymptotic variance-covariance of the vector $\hat{Z}$ that stacks all estimates $\left(\hat{Z}_{c}^{I J}\right)$. Note that by construction, the elements of $\Omega$ that relate to different cohorts are all zero: $\Omega$ is a collection of $T$ square matrices of dimension $\mathcal{K}^{2}$, which we denote $\Omega_{c}$. To obtain a minimum distance estimator of 
the parameters $A_{I J}, B_{c}^{I}$ and $C_{c}^{J}$, we will simply choose $\left(A_{I J}, B_{c}^{I}, C_{c}^{J}\right)$ so as to minimize

$$
\sum_{c=1}^{T} \varepsilon_{c}^{\prime} W_{c} \varepsilon_{c}
$$

where $\varepsilon_{c}=\hat{Z}_{c}^{I J}-A_{I J}-B_{c}^{I}-C_{c}^{J}$ and the positive definite matrices $W_{c}$ are well-chosen.

This is simply minimum distance estimation, with the $Z_{c}^{I J}$ as "auxiliary parameters" and the $A_{I J}, B_{c}^{I}$ and $C_{c}^{J}$ as "parameters of interest". From the general theory of MDE it follows that under the null (mixed) hypothesis, the resulting estimators are consistent and asymptotically normal. Their precision is highest when $W_{c}$ converges to $\Omega_{c}^{-1}$ as the sample size gets large; and we can then test for the null hypothesis using as test statistic the minimized value of the criterion in (38). Let $n_{Z}$ be the number of auxiliary parameters (that is, $n_{Z}=C \mathcal{K}^{2}$ ) and $n_{r}$ be the number of parameters of interest. Then under the null this test statistic is distributed asymptotically as a $\chi^{2}\left(n_{Z}-n_{r}\right)$. It is easy to see that for the benchmark model,

$$
n_{r}=\mathcal{K}^{2} T-\mathcal{K}^{2}-(2 \mathcal{K}-1)(T-1)
$$

and the test has

$$
\mathcal{K}^{2}+(2 \mathcal{K}-1)(T-1)
$$

degrees of freedom. For whites for instance, $\mathcal{K}=5$ and $T=28$. The unconstrained model has 700 degrees of freedom, and the benchmark model only has 268. Under the null, the test statistic should be approximately drawn from a $\chi^{2}(432)$.

In practice, within each cohort $c$ we use the estimated covariance matrix of the estimated matching probabilities to compute an estimate of the matrix $\Omega_{c}$ (see Appendix B for details.) Minimizing the expression in (38) is very simple since the $\varepsilon_{c}$ terms are linear in the unknown parameters. The only (minor) difficulty is to keep track of the necessary location normalizations.

\subsection{The extensions}

\subsubsection{Changing complementarities}

The models with time-varying complementarities of section 4.2 .1 can be dealt with in exactly the same way, and so can the models with preferences for age differences of section 4.2.3. Only the numbers of degrees of freedom of the $\chi^{2}$ differ, because these extensions have more parameters, and because (for the model with age differences) we fit more margins. 


\subsubsection{The Heteroskedastic Model}

Introducing heteroskedasticity as in section 4.2 .2 only complicates inference slightly. With time-invariant heteroskedasticity, our basic identity now is

$$
Z_{I J}^{c}=\sigma^{I} \log \frac{\operatorname{Pr}(J \mid I, c)}{\operatorname{Pr}(0 \mid I, c)}+\mu^{J} \log \frac{\operatorname{Pr}(I \mid J, c)}{\operatorname{Pr}(0 \mid J, c)} .
$$

First assume that complementarities are constant across cohorts. Then the null hypothesis is:

$$
\left(H_{0}\right): \exists\left(\sigma_{I}, \mu_{J}, A_{I J}, B_{c}^{I}, C_{c}^{J}\right) \text { s.t. } \sigma^{I} \log \operatorname{Pr}(J \mid I, c)+\mu^{J} \log \operatorname{Pr}(I \mid J, c)=A_{I J}+B_{c}^{I}+C_{c}^{J} .
$$

Note that this involves $(2 \mathcal{K}-1)$ additional parameters (we need to keep one of the $\sigma, \mu$ 's equal to one.)

The more important change is that the asymptotic covariance matrix $\Omega_{c}$ now involves the unknown parameters $\sigma_{I}$ and $\mu_{J}$. But we are especially interested in testing the null hypothesis of homoskedasticity (our Choo and Siow benchmark) within the broader hypothesis $\left(H_{0}\right)$. Alain Monfort and Roger Rabemananjara (1990) show that this can be done by simply

1. choosing $W_{c}$ as we did for the benchmark model;

2. minimizing

$$
\sum_{c=1}^{C} \varepsilon_{c}^{\prime} W_{c} \varepsilon_{c}
$$

where this time $\varepsilon_{c}^{I J}=\sigma^{I} \log \widehat{\operatorname{Pr}}(J \mid I, c)+\mu^{J} \log \widehat{\operatorname{Pr}}(I \mid J, c)-A_{I J}-B_{c}^{I}-C_{c}^{J}$ and we minimize over the $(\sigma, \mu, A, B, C)$ parameters

3. and subtracting the value of this minimized criterion from that of the minimized benchmark criterion.

Under the null of homoskedasticity, the resulting statistic is a difference of two $\chi^{2}$ statistics. The results in Monfort-Rabemananjara show that these two $\chi^{2}$ statistics are asymptotically independent, so that their difference also follows a $\chi^{2}$, with $(2 \mathcal{K}-1)$ degrees of freedom here.

\section{Results}

\subsection{Investment in children's human capital}

It is clear that investments in children's human capital, particularly at a younger age, are more important than ever; several authors have written extensively on the topic ${ }^{25}$. Let us start with our first set of predictions, relative to time spent on various domestic and children-related activities. Table 1, borrowed

${ }^{25}$ See for instance Heckman $(2006,2011,2014)$. 
from Browning, Chiappori and Weiss (2015), gives the evolution of time spent by spouses on chores and child care over three decades in the US, as well as Canada and the UK. Two patterns emerge from these data. First, the time women spend on chores decreases significantly, while the time spent by men increases slightly. This pattern fully supports the assumption of substituability between male and female time; and it is consistent with the increase in female opportunity cost. Second, the time women spend with children has increased (by 65 to $75 \%$, depending on children's age, in the US); and men are spending much more time with chidren than they used to-about three times more in the US. This is exactly in line with the predictions we derived earlier.

[Table 1 about here.]

A second prediction was that this evolution should be stronger among more educated couples. The following graph is based on data in Altintas (2016); it plots the average time spent daily in developmental child care activities by parental education. While an increase can be seen at all education levels, it is much larger for parents with college education and above.

[Figure 14 about here.]

Next, we come to the core of our contribution; that is, we test and estimate the matching models described above.

\subsubsection{Tests of the benchmark model}

We start with the benchmark model, tested on the white population. Recall our prediction that each of $16 T$-dimensional vectors $\boldsymbol{d}^{\boldsymbol{I} \boldsymbol{J}, \boldsymbol{K} \boldsymbol{L}}=\left(d_{c}^{I J, K L}, c=1, \ldots, T\right)$ be constant. These requirements can readily be checked on the data. Here we take reference categories to be $K=L=3$ and we plot the $d_{c}^{I J, 33}$ demeaned over cohorts. Figure 15 shows the graphs corresponding to the "diagonal" elements $d_{c}^{I I, 33}, I=1,2,4,5$ of assortatively matched white couples. Under the null, the blue curve (and the dashed smoothed blue curve) should be identically 0 ; the dotted curves give the $95 \%$ confidence band. The property is clearly violated for college and college-plus educated pairs, for which the trend is clearly ascending. This suggests an increase in assortativeness, at least for the more educated fraction of the population.

Altogether, the graphs suggest that the benchmark model is rejected by the data. The formal test described in section 5.1 has 432 degrees of freedom, and gives a $\chi^{2}$ statistic of 1573.7 , way above the $5 \%$ critical value of 481.5 (the $p$-value is $2 e-129$.)

We also estimate and test the version allowing for age differences. The conclusions are similar: when we average errors over age differences as discussed above, the $\chi^{2}$ statistic has value 1526.5 with 405 degrees of freedom, while the $5 \%$ critical value is 452.9 , leading to a $p$-value that the computer cannot distinguish from zero.

[Figure 15 about here.] 
[Figure 16 about here.]

Our findings are totally different for black couples. Given the much smaller sample size, especially for higher education, we only use four education categories, aggregating college and college-plus. Figure 16 shows little evidence for deviations from the benchmark model. The formal test confirms this visual impression. The number of degrees of freedom is now 198, leading to a $5 \%$ critical value of 231.8. The $\chi^{2}$ statistic is 185.9 , which corresponds to a $p$-value of 0.72 . We therefore fail to reject the null that preferences for assortativeness have not changed over the period among the African-American population.

Our test of course has less power on a smaller sample; and there are twenty times fewer black couples than white couples in our data. For any given deviation from the null hypothesis, the $\chi^{2}$ statistic is known to scale linearly with sample size. Multiplying the value of the $\chi^{2}$ statistic for black couples by 20 would lead to a clear rejection of the null. However, the value of the $\chi^{2}$ statistic we obtained in an earlier version of the paper with five rather than seven waves of the ACS was actually larger. Figure 16 also shows no clear pattern of deviations over time, unlike Figure 15. This suggests to us that our result for black couples is not simply due to low power.

\subsubsection{White sample: extensions}

[Figure 17 about here.]

Since the benchmark model is rejected for the white population, we next consider the linear and quadratic extensions. Figure 17 gives, again for diagonal pairs $(I, I)$, the standardized residuals of three regressions of the $d_{c}^{I I, 33}$, corresponding respectively to the benchmark model, the linear trend, and the quadratic trend extensions. Since the variance is normalized to be 1 , theory would require these residuals to remain between -1.96 and 1.96 for $95 \%$ of observations. The linear trend models appear to fit data significantly better than the benchmark. Moreover, while the quadratic component further increases the fit, its contribution is much more incremental.

While the model is still rejected, the rejection is much less drastic than previously. Specifically, the $\chi^{2}$ statistic is 677.7 , while the $5 \%$ critical value (for 416 degrees of freedom) is 464.6 . The spectacular decrease in the $\chi^{2}$ statistic indicate that preferences did change over the period; an exact description of these changes is provided in the next subsection. Under the quadratic version, the number of degrees of freedom is now 400 , giving a $5 \%$ critical value of 447.6 ; the test statistic equals 527.9. The null that all quadratic terms are zero is rejected. The test using age differences gives very similar results.

[Table 2 about here.]

We therefore conclude that in the white population, the surplus generated by assortativeness changed significantly over the period. The direction of these changes is quite interesting. Remember from section 4.2.1 that these changes 
push towards more assortativeness if and only if the matrix $\boldsymbol{b}$ formed by the coefficients of the linear trends in (34) is supermodular on the diagonal; that is iff each $D^{I I, K K} \boldsymbol{b}$ is positive when $1 \leq I<K$. There are 10 such numbers; and our results, as shown in Table 2, are unambiguous. Of these 10 numbers, 7 are significantly different from zero (despite the fact that, in the end, these coefficients represents third differences); strikingly, all are positive. A formal test of the null hypothesis that these numbers are zero (the least-favorable case for our predictions) confirms this conclusion. If we only use the three values generated from the restricted submatrix with $3 \leq I<K$, the evidence for changes towards more assortativeness at the top of the distribution of human capital is overwhelming ${ }^{26}$.

All in all, our findings provide a strong confirmation to our basic prediction. For the more educated part of the sample, the supermodularity of the $Z$ matrix has increased over time, indicating higher gains from assortativeness. Decreases in supermodularity are actually observed, but only for couples at the bottom of the distribution and where the wife is less educated than the husband.

\subsubsection{Heteroskedasticity}

We next test for heteroskedasticity along the lines suggested in 4.2.2. Remember that our test statistic is the difference of two $\chi^{2}$ statistics, one of which is computed over the best heteroskedastic model. Unfortunately, we haven't been able to estimate a fully heteroskedastic model: some of the $\mu^{J}$ 's (the dispersion parameters for women) go to the lower bound of zero. We settled on an approach in which we only allow the dispersions for one gender to deviate from the value of one. These two semi-heteroskedastic models converged without difficulty. The evidence for hetoroskedasticity is strong, with $p$-values smaller than $1 e-$ 30 for both genders. However, allowing for this semi-heteroskedasticity does not improve the fit of the model much: the $\chi^{2}$ statistic only decreases from its "homoskedastic" value of 1573.7 to 1390 for the men-heteroskedastic and 1414 for the women-heteroskedastic models. The data still spectacularly rejects models with constant complementarities.

A heteroskedastic model with time trends affecting the supermodular core is in principle identifiable; but in practice the empirical estimates are not robust, possibly indicating an overparametrization. Given our emphasis on the evolution of assortativeness across cohorts, we keep the trends but omit heteroskedasticity in what follows.

\footnotetext{
${ }^{26}$ We base our tests on the minimum of the standardized values of the estimated $D^{I I, K K} \boldsymbol{b}$; and we simulate the one-sided $5 \%$ critical value under the null using the estimated covariance matrix. A "minimum statistic" smaller than the critical value would reject the hypothesis that the matrix $b$ is supermodular on the diagonal. The statistic on the 10 numbers equals -1.808 , with a critical value of -2.54 ; the $p$-value of the test is 0.73 . The minimum statistic is 4.44 for the restricted submatrix, while the critical value is -2.06 . The $p$-value of the test is indistinguishable from 1 .
} 


\subsection{The marital college premium: the African-American sample}

Since the benchmark model is not rejected for Afro-Americans, we can directly analyze its implications. For any education group, we define the "marital education premium" as the gain in expected utility an individual would obtain on the marriage market if this individual had the level. For a man of group $I<\mathcal{K}$, for instance, the education premia is simply $\left(\bar{u}^{I+1}-\bar{u}^{I}\right)$.

Figures 18 and 19 show the evolution of our estimated male and female marital education premia for blacks. The plots are noisy, for college-educated men in particular; and even one standard-error confidence bands are wide. Still, none of these lines seem to be sloping up or down. Fitting linear trends confirms the visual impression. The marital value of acquiring a high-school degree has a significant negative trend for both men and women, and the marital value of acquiring a college degree has a significant positive trend for women; but they are very small, as the scales suggest.

To rephrase this: the marital education premia stayed almost constant for black men as well as for black women. In particular, the additional marital expected utility from a college degree hardly changed over the period for either gender.

[Figure 18 about here.]

[Figure 19 about here.]

This finding can be related to the specific evolution of the demand for higher education among African-Americans over the period: as discussed earlier, no significant difference appears between men and women. While a precise investigation of the causes for that difference is beyond the scope of this paper, differences in investment in children education may be one of them. In a recent contribution, Gayle, Golan and Soytas (2014) show for instance that married black women spend less time with children than their white counterparts, despite little difference in education level and only a slightly larger number of children $^{27}$. This difference can be explained in a number of different ways. It may reflect differences in the costs of education perceived by black and whites, as suggested by Neal (2006) in his extensive survey of the topic ${ }^{28}$; or it may re-

\footnotetext{
27 "[...] Married black females on average have slightly more children than married white females (1.43 for blacks versus 1.27 for whites) but spend 30 percent less time with young children than married white females. [...] Thus in terms of demographic characteristics, black and white married females are similar but black married females spend less time with young children." (Gayle et al 2015, p. 7).

28 "[...] there is some evidence that blacks attend less effective schools than white students, and although this racial gap in school quality cannot be linked to racial differences in school funding levels, it may indicate that blacks pay higher implicit, if not explicit, costs to attend quality schools" (p. 562), and "[...] the fact that parenting behaviors differ greatly by race among families that are similar with respect to wealth, neighborhood quality, family structure, and measured maternal human capital raises the possibility that norms concerning child rearing differ among blacks and whites in important ways" (p. 568).
} 
flect a less precise knowledge of the production function of child human capital, as argued by several authors ${ }^{29}$. This remains an underinvestigated question.

\subsection{The white population}

\subsubsection{The marital college premium: evolution over cohorts}

Figures 20 and 21 represent our estimated marital education premia for white men and women. Two facts stand out. First, the marital premia of a college education (SC, CG, or CG+) increase over time. Second, and more interestingly for our purpose, male and female profiles are dissimilar.

[Figure 20 about here.]

[Figure 21 about here.]

For men, the graph of the marital education premium for the highest levels of education - college-plus - is rather flat: the "college-plus premium", defined as the difference in marital utility between college and college-plus, is roughly constant over the period.

For women, however, the picture is very different. While women of older cohorts had negative marital college premia, they have become positive for recent cohorts. Even more interesting is the reduction of the difference between college and college-plus women, which translates into a notable increase in the college-plus premium for women.

As a result of these contrasted findings for men and women, the (double) difference between female and male marital college-plus premia increases significantly over the period. Using the same notation as above, if $\bar{u}_{c}^{I}$ (resp. $\bar{v}_{c}^{I}$ ) denotes the expected marital gain for men (women) with education $I$ in cohort $c$, we consider the evolution of the double difference

$$
\Delta_{c}^{I}=\left(\bar{v}_{c}^{I+1}-\bar{v}_{c}^{I}\right)-\left(\bar{u}_{c}^{I+1}-\bar{u}_{c}^{I}\right)
$$

over cohorts. For $I=4$ for instance, $\bar{u}_{c}^{I+1}-\bar{u}_{c}^{I}$ thus represents the college-plus premium for men, and $\Delta_{c}^{I}$ is the difference in the college-plus premia of women and men. Our prediction of a significant asymmetry between the respective evolutions of male and female college-plus premia should translate into a increase in this double difference over time. Figure 22 tests this by plotting the estimated $\Delta_{c}^{I}$, with $95 \%$ error bars and a smoother. The increase in $\Delta_{c}^{4}$ over time is highly significant, both in statistical and economic terms. In contrast, $\Delta_{c}^{1}$ and $\Delta_{c}^{3}$ show no significant change. The transition from high-school graduate to "some college", as represented by $\Delta_{c}^{2}$, also shows some increase over time, but at as slower rate.

[Figure 22 about here.]

${ }^{29}$ See for instance Cunha et al. (2013). 
In summary, we see a significant increase in the college-plus premium for women, whereas no such evolution is apparent for men or for other educational transitions. It bears repeating that this conclusion obtains from the sole analysis of matching patterns over time, without any other data on intrahousehold allocations. It nevertheless strongly supports CIW's suggestion that the increase in female demand for higher education could be motivated in part by the divergences between the respective evolutions of male and female marital college-plus premia. ${ }^{3031}$

\subsubsection{Decomposition}

The marital college premium can be decomposed into several components. First, education affects the probability of being married. Second, conditional on being married, it also affects the education of the spouse (or more exactly its probability distribution); intuitively, we expect educated women to find a "better" husband, at least in terms of education, and conversely. Third, the impact on the total surplus generated by marriage is twofold. Take women for instance. A wife's education has a direct impact on the surplus; this impact can be measured, for college-plus education, by the difference $\left(Z_{c}^{I 5}-Z_{c}^{I 4}\right)$. In addition, since a more educated woman is more likely to marry a more educated husband, the husband's higher expected education further boosts the surplus, by the average of the $\left(Z_{c}^{5 J}-Z_{c}^{4 J}\right)$ terms weighted by the difference in probability of marrying a college-plus educated husband instead of a college graduate. Finally, the share of the surplus going to the wife in any given match is also affected by her education. This share is given by the ratio

$$
\frac{\bar{v}_{c}^{J}}{\bar{u}_{c}^{I}+\bar{v}_{c}^{J}}
$$

for a woman of education $J$ who marries a man of education $I$.

[Table 3 about here.]

[Table 4 about here.]

[Table 5 about here.]

All these components are readily computed from our estimates; they are summarized in Tables 3 to 7 . The first three tables summarize patterns that we

\footnotetext{
${ }^{30}$ As noted before, a limitation of our analysis is the lack of adequate data on divorce. The study by Schwartz and Han (2014) reports that for recent cohorts, being more educated than the husband is no longer a handicap for women in terms of divorce probability. Although based on very small samples, this finding is consistent with the rising incentive of women to invest in schooling that our model predicts.

${ }^{31} \mathrm{~A}$ possible concern is related to the frictionless nature of our setting. If, in practice, individuals are more likely to meet potential mates who belong to the same education class, then an increase in the population of educated women might in principle have a mechanical impact on matching patterns independently of the evolution of the surplus. However, recent work by Jaffe and Weber (2016) argue that this cannot be the case.
} 
already discussed in the introduction. The marriage probabilities decline, very sharply for less educated individuals, and much less for college-educated women especially. Educated men are more likely to marry an educated wife than they used to. The pattern is opposite for college-educated women; in partiuclar they are much less likely to marry a college-plus husband.

[Table 6 about here.]

[Table 7 about here.]

More interesting are the last two tables, which are specific to our structural approach. The joint surplus declines in 17 of the 25 cells. Of the eight positive cells (in bold in Table 6), seven concern couples formed by college or collegeplus educated women; and in all of these eight cells, the wife is at least as educated as the husband. The decline in surplus is always smaller (or inverted) for more educated couples, which is consistent with the increased preferences for assortativeness at the top of the distribution.

Lastly, the intra-household distribution of the surplus also varies significantly, and with a striking pattern: the share of the wife increases in matches in which she at least as well-educated as her husband. This corresponds to the cells on and above the diagonal in Table 7. One can go further: the gains in surplus sharing of the wives are strongest in couples with a large educational advantage of the wife. On the other hand, highly educated men who continue to "marry down" have gained a larger share of a joint surplus which has become smaller over the years, as shown in Table 6 .

\section{Conclusion}

It has long been recognized (at least since Becker's 1973 seminal contributions) that the division of the surplus generated by marriage should be analyzed as an equilibrium phenomenon. As such, it responds to changes in the economic environment; conversely, investments made before marriage are partly driven by agents' current expectations about the division of surplus that will prevail after marriage. Theory shows that such considerations may explain the considerable differences in male and female demands for higher education. When deciding whether to go to college, agents take into account not only the labor market college premium (i.e., the wage differential resulting from a college education) but also the "marital college premium", which represents the impact of education on marital prospects; the latter includes not only marriage probabilities, but also the expected "quality" of the future spouse and the resulting size and distribution of the marital surplus. While the first aspect - the labor market college premium - has been abundantly discussed, the second - the marital college premium - has been all but ignored. We argued in this paper that this omission may have severely hampered our understanding of recent evolutions in the demand for higher education. 
We provided a simple but rich model in which the marital college premium can be econometrically identified. Our framework generalizes the original contribution by Choo and Siow (2006); it can be (over)identified using temporal variations in the compositions of the populations. Applying the model to US data, we find gender- and race-specific evolutions. There is no evidence of changes in preference for assortativeness among the black population; among whites, on the contrary, we find a highly significant increase, particularly among the most educated subsample. The evolution of the marital college and college-plus premium is similar across genders for the African-American population; this sharply contrasts with the white sample, where the marital college-plus premium increases much more for women that for men. All in all, our findings support the claim that the marriage market has played an important role in the demand for higher education in the recent decades.

We claim that these evolutions can be explained by basic economic arguments. The value of investment in children's human capital has drastically increased over the period, while the amount of time available was boosted by technological innovations that freed households - mostly women - from spending much of their time on basic chores. A first consequence has been a drastic change in time allocation patterns: less time was spent on chores and much more on children. As theory predicts, these shifts have resulted in significant changes in matching patterns. They can explain in particular the significant increase in assortative matching that several authors observed - and that our more structural estimation confirms - at the top of the human capital distribution. This impact was amplified by the self-reinforcing nature of the equilibrium. An initial impulse affecting a gender's stock of human capital, added to a slight difference in initial ability (as described by Gary Becker, William Hubbard and Kevin Murphy 2010) results in higher incentives to invest in education, and ultimately in significant changes in both education profiles and marital patterns.

All in all, this period exhibits a boost in returns to human capital investments, which resulted not only in higher incentives to invest, but in a general reshuffling of equilibrium patterns on the marriage market. Our belief is that explicit modelling can help shed light on these complex mechanisms. 


\section{References}

Albanesi, S. and C. Olivetti (2016), "Gender Roles and Medical Progress", Journal of POlitical Economy, 124, 650-695.

Altintas, E. (2016), "The Widening Education Gap in Developmental Child Care Activities in the United States, 1965-2013", Journal of Marriage and Family 78, 26-42.

Autor, D. (2014), "Skills, education, and the rise of earnings inequality among the "other 99 percent"", Science, 344, 843-851.

Becker, G. (1973), "A Theory of Marriage: Part I", Journal of Political Economy, 81, 813-846.

Becker, G. (1974), "A Theory of Marriage: Part II", Journal of Political Economy, 82, S11-S26.

Becker, G. (1991), Treatise on the family, Cambridge Mass: Harvard University Press.

Becker, Gary S., William H. J. Hubbard, and Kevin M. Murphy (2010), "The Market for College Graduates and the Worldwide Boom in Higher Education of Women", American Economic Review, 100(2): 229-33.

Bergstrom, T. and R. Cornes (1983), "Independence of Allocative Efficiency from Distribution in the Theory of Public Goods", Econometrica, 51, 1753-1765.

Botticini, M., and A. Siow (2008), "Are there Increasing Returns in Marriage Markets?", University of Toronto, mimeo.

Bronson, M. A. (2014), "Degrees Are Forever: Marriage, Educational Investment, and Lifecycle Labor Decisions of Men and Women", working paper, UCLA.

Browning, M., P.-A. Chiappori, and Y. Weiss (2014), Economics of the Family. Cambridge University Press, New York.

Bruze, G., Svarer M. and Y. Weiss (2015), "The Dynamics of Marriage and Divorce" , Journal of Labor Economics, 33, 123-170.

Chiappori, PA (2017), Matching with Transfers, Princeton University Press, Princeton, forthcoming.

Chiappori, PA, M. Iyigun and Y. Weiss (2009), "Investment in Schooling and the Marriage Market", American Economic Review, 99, 1689-1714.

Chiappori, PA, R. McCann and L. Nesheim (2010) "Hedonic Price Equilibria, Stable Matching, And Optimal Transport: Equivalence, Topology, And Uniqueness", Economic Theory, 42 2, 317-354.

Chiappori, PA, and B. Salanié (2016), "The Econometrics of Matching Models", Journal of Economic Literature, forthcoming.

Cherlin, A. (1992) Marriage, Divorce, Remarriage, Cambridge Mass: Harvard University Press.

Choo, E. and A. Siow (2006), "Who Marries Whom and Why", Journal of Political Economy, 114, 175-201. 
Cunha, Flávio, Irma Elo and Jennifer Culhane (2013), 'Eliciting Maternal Expectations about the Technology of Cognitive Skill Formation', NBER Working Paper 19144.

Dagsvik, J.K. (2000), "Aggregation in Matching Markets" International Economic Review, 41, 27-57.

Del Boca, Daniela, Christopher Flinn and Matthew Wiswall (2014), "Transfers to Households with Children and Child Development", forthcoming, Economic Journal.

Doepke, M. and M. Tertilt (2009), "Womenâ's Liberation: Whatâ's in It for Men?", Quarterly Journal of Economics, 124, 1541-91.

Doepke, M., M. Tertilt and A. Voena (2011), "The Economics and Politics of Womenâ's Rights", NBER Working Paper 17672.

Fox, J. (2010a), "Identification in Matching Games", Quantitative Economics, 1, 203-254.

Fox, J. (2016), "Estimating matching games with transfers", Rice University mimeo.

Fox, J., D. Hsu and C. Yang (2015), "Unobserved Heterogeneity in Matching Games with an Application to Venture Capital", University of Michigan mimeo.

Galichon, A., and B. Salanié (2015), "Cupid's Invisible Hand: Social Surplus and Identification in Matching Models", Columbia University mimeo.

Goldin, C. and Katz, L. (2002), "The power of the pill: oral contraceptives and women's career and marriage decisions" , Journal of Political Economy, 110, 730-770.

Goldin, C. and Katz, L. (2008), The Race Between Education And Technology, Harvard University Press, Cambridge.

Goldin, Claudia and Lawrence F. Katz (2009), 'The Future of Inequality: The Other Reason Education Matters So Much', Aspen Institute Congressional Program 24(4): 7-14. Education Reform: Sixteenth Conference, August 17-August 22, 2009. Washington, DC: Aspen Institute. Previously published in Milken Institute Review, 3rd Q 2009.

Graham, B. (2011), "Econometric Methods for the Analysis of Assignment Problems in the Presence of Complementarity and Social Spillovers" , in J. Benhabib, M. Jackson and A. Bisin eds: Handbook of Social Economics, North-Holland, Vol. 1B, 965-1052.

Greenwood, Jeremy, Nezih Guner, Georgi Kocharkov and Cezar Santos (2014), "Marry Your Like: Assortative Mating and Income Inequality", NBER Working Paper No. 19829, NBER

Greenwood, J., A. Seshadri and M. Yorukoglu (2005), "Engines of Liberation," Review of Economic Studies, 72, 109-133.

Gullickson, A. (2006), "Black/White Interracial Marriage Trends, 18502000", Journal of Family History, 32, 289-312.

Heckman, J. J. (2006), "Skill formation and the economics of investing in disadvantaged children", Science, 312, 1900-1902.

Heckman, J. J. (2011), "The Economics of Inequality: the Value of Early Childhood Education", American Educator, 31-47. 
Heckman, J. J. (2014), "Invest in early childhood development: Reduce deficits, strengthen the economy.", http://heckmanequation.org.

Jaffe, S., and S. Weber (2016), "Idiosyncratic Preferences and Limited Choice Sets in Matching", Working paper, University of Chicago.

Lundberg, S. and R. Pollak (2013), "Cohabitation and the Uneven Retreat from Marriage in the U.S., 1950-2010", NBER Working Paper 19413.

McFadden, D., (1973), "Conditional Logit Analysis of Qualitative Choice Behavior," in P. Zarembka (ed.), Frontiers in Econometrics, 105-142, Academic Press: New York.

Michael, R., (2000), "Abortion decisions in the U.S" , in Laumann, E, Michael, R (eds.), Sex, Love and Health: Public and Private Policy, University of Chicago Press.

Mishel, L., J. Bivens, E. Gould and H. Shierholz (2012), The State of Working America, 12th Edition, Cornell University Press, Ithaca.

Monfort, A. and and R. Rabemananjara (1990), "From a Var Model to a Structural Model, with an Application to the Wage-Price Spiral", Journal of Applied Econometrics, 5, 203-227.

Mourifié, I. and A. Siow (2014), "Cohabitation versus marriage: Marriage matching with peer effects", University of Toronto mimeo.

Neal, D. and A. Rick (2014), "The Prison Boom and the Lack of Black Progress After Smith and Welch", NBER Working Paper 20283.

Ruggles, S., K. Genadek, R. Goeken, J. Grover, and M. Sobek (2015), "Integrated Public Use Microdata Series: Version 6.0 [Machine-readable database]". Minneapolis: University of Minnesota.

Schoen, R. and J. Klugel (1988), "The Widening Gap in Black and White Marriage Propensities", American Sociological Review, 53, 895-907.

Schwartz, C. and Han, H. (2014) "The Reversal of the Gender Gap in Education and Trends in Marital Dissolution " Demography, 42, 621-646.

Schwartz, C. and Mare, R. (2005), "Trends in Educational Assortative Marriage from 1940 to 2003" , American Sociological Review, 79, 605-629.

Shapley, L. and M. Shubik (1972), "The Assignment Game I: The Core", International Journal of Game Theory, 1, 111-130.

Siow, A. (2015), "Testing Becker's Theory of Positive Assortative Matching", Journal of Labor Economics, 33, 409-441.

Siow, A., and E. Choo (2006), "Estimating a Marriage Matching Model with Spillover Effects," Demography, 43, 463-490.

Seitz, S. (2009), "Accounting for Racial Differences in Marriage and Employment," Journal of Labor Economics, 27, 385-437.

Stevenson, B. and J. Wolfers (2007), "Marriage and Divorce: Changes and their Driving Forces", Journal of Economic Perspectives, 21, 27-52.

Weiss, Y. and R. Willis (1997), "Match Quality, New Information, and Marital Dissolution" , Journal of Labor Economics, 15, 293-329.

Wilson, W. J., and K. Neckerman (1986), "Poverty and family structure: The widening gap between evidence and public policy issues", in S. H. Danziger \& D. H. Wienberg (Eds.), Fighting poverty: What works and what doesn't, Cambridge, MA: Harvard University Press. 


\section{A Proof of Proposition 2}

The proof is in several steps. Let $(i \in I, j \in J)$ be a matched couple. Then:

1. First, man $i$ must better off than being single, which gives:

$$
U^{I J}+\alpha_{i}^{I J}-\alpha_{i}^{I \emptyset} \geq 0
$$

hence $\alpha_{i}^{I J}-\alpha_{i}^{I \emptyset} \geq-U^{I J}$ and the same must hold with woman $j$.

2. Take some woman $j^{\prime}$ in $J$, currently married to some $i^{\prime}$ in $I$. Then $i$ must be better off matched with $j$ than $j^{\prime}$, which gives:

$$
\begin{aligned}
U^{I J}+\left(\alpha_{i}^{I J}-\alpha_{i}^{I \emptyset}\right) & \geq z_{i j^{\prime}}-v_{j^{\prime}} \\
& =Z^{I J}+\left(\alpha_{i}^{I J}-\alpha_{i}^{I \emptyset}\right)+\left(\beta_{j}^{I J}-\beta_{j}^{\emptyset J}\right)-\left(V^{I J}+\left(\beta_{j^{\prime}}^{I J}-\beta_{j^{\prime}}^{\emptyset J}\right)\right)
\end{aligned}
$$

and one can readily check that this inequality is always satisfied as an equality, reflecting the fact that $i$ is indifferent between $j$ and $j^{\prime}$, and symmetrically $j$ is indifferent between $i$ and $i^{\prime}$.

3. Take some woman $k$ in $K \neq J$, currently married to some $i^{\prime}$ in $I$. Then " $i$ is better off matched with $j$ than $k$ " gives:

$$
U^{I J}+\left(\alpha_{i}^{I J}-\alpha_{i}^{I \emptyset}\right) \geq z_{i k}-v_{k}=Z^{I K}+\left(\alpha_{i}^{I J}-\alpha_{i}^{I \emptyset}\right)+\beta_{k}^{I K}-\left(V^{I K}+\beta_{k}^{I K}\right)
$$

which is equivalent to

$$
\alpha_{i}^{I J}-\alpha_{i}^{I K} \geq U^{I K}-U^{I J}
$$

and we have proved that the conditions (19) are necessary. The proof is identical for $(20)$.

4. We now show that these conditions are sufficient. Assume, indeed, that they are satisfied. We want to show two properties. First, take some woman $j^{\prime}$ in $J$, currently married to some $l$ in $L \neq I$. Then $i$ is better off matched with $j$ than $j^{\prime}$. Indeed,

$$
\begin{aligned}
U^{I J}+\left(\alpha_{i}^{I J}-\alpha_{i}^{I \emptyset}\right) & \geq z_{i j^{\prime}}-v_{j^{\prime}} \\
& =Z^{I J}+\left(\alpha_{i}^{I J}-\alpha_{i}^{I \emptyset}\right)+\left(\beta_{j}^{I J}-\beta_{j}^{\emptyset J}\right)-\left(V^{I J}+\left(\beta_{j^{\prime}}^{I J}-\beta_{j^{\prime}}^{\emptyset J}\right)\right)
\end{aligned}
$$

is a direct consequence of (20) applied to $l$. Finally, take some woman $k$ in $K \neq J$, currently married to some $l$ in $L \neq I$. Then $i$ is better off matched with $j$ than $j^{\prime}$. Indeed, it is sufficient to show that

$$
U^{I J}+\alpha_{i}^{I J} \geq z_{i k}-v_{k}=Z^{I K}+\left(\alpha_{i}^{I J}-\alpha_{i}^{I \emptyset}\right)+\beta_{k}^{I K}-\left(V^{I K}+\beta_{k}^{I K}\right)
$$


But from (20) applied to $k$ we have that:

$$
\beta_{k}^{L K}-\beta_{k}^{I K} \geq V^{I K}-V^{L K}
$$

and from (19) applied to $i$ :

$$
\alpha_{i}^{I J}-\alpha_{i}^{I K} \geq U^{I K}-U^{I J}
$$

and the required inequality is just the sum of the previous two.

\section{B Estimating the variance-covariance matrix $\Omega_{c}$}

Our tests rely on an estimate $\hat{\Omega}_{c}$ of the variance-covariance matrix of the estimated surplus matrix $\left(Z_{c}^{I J}\right)_{I, J}$. Recall equation (37):

$$
\hat{Z}_{c}^{I J}=\log (\widehat{\operatorname{Pr}}(J \mid I, c))+\log (\widehat{\operatorname{Pr}}(I \mid J, c)) .
$$

When constructing this estimate, we take into account the sampling weights in the American Community Survey. To estimate $\widehat{\operatorname{Pr}}(J \mid I, c)$, we take a ratio:

- the numerator is the sum of the sampling weights $w_{h}$ of the households that correspond to a married couple in which the husband belongs to group $I$ and cohort $c$, and the wife belongs to group $J$

- the denominator is the sum of the sampling weights of all households in which the man belongs to group $I$ and cohort $c$.

That is, in hopefully transparent notation,

$$
\widehat{\operatorname{Pr}}(J \mid I, c)=\frac{\sum_{h} w_{h} \mathbb{1}(h \supseteq I, J, c)}{\sum_{h} w_{h} \mathbb{1}(h \supseteq I, c)} .
$$

where the sum is over all households $h$ in our sample.

Similarly, we estimate $\widehat{\operatorname{Pr}}(I \mid J, c)$ by $\sum_{h} w_{h} \mathbb{1}(h \in I \cap J) / \sum_{h} w_{h} \mathbb{1}(h \in I)$.

Note that as explained in the text, we take women to be one year younger, to allow for the modal age difference. An incidental advantage of this procedure is that it guarantees that our estimates of $\widehat{\operatorname{Pr}}(J \mid I, c)$ and of $\widehat{\operatorname{Pr}}(I \mid J, c)$ are statistically independent. As a consequence,

$$
\begin{aligned}
\Omega_{c}(I J, K L) & =\operatorname{cov}\left(\hat{Z}_{c}^{I J}, \hat{Z}_{c}^{K L}\right) \\
& =\operatorname{cov}(\widehat{\operatorname{Pr}}(J \mid I, c), \widehat{\operatorname{Pr}}(L \mid K, c))+\operatorname{cov}(\widehat{\operatorname{Pr}}(I \mid J, c), \widehat{\operatorname{Pr}}(K \mid L, c)) .
\end{aligned}
$$

Moreover, in large markets the estimated matching patterns of men of two different groups $I$ and $K$ are also statistically independent, as are the estimated matching patterns of women of two different groups $J$ and $L$. Denoting $V_{I c}^{m}$ 
(resp. $V_{J c}^{w}$ ) the asymptotic variance-covariance matrix of the estimated probabilities $\widehat{\operatorname{Pr}}(\cdot \mid I, c)$ (resp. $\widehat{\operatorname{Pr}}(\cdot \mid J, c))$ for each $(J, c)$ (resp. for each $(I, c)$ ), we obtain

$$
\Omega_{c}(I J, K L)=\mathbb{1}(I=K) \frac{V_{I c}^{m}(J, L)}{\operatorname{Pr}(J \mid I, c) \operatorname{Pr}(L \mid I, c)}+\mathbb{1}(J=L) \frac{V_{J c}^{w}(I, K)}{\operatorname{Pr}(I \mid J, c) \operatorname{Pr}(K \mid J, c)} .
$$

To turn this equation into an estimator, we plug in our estimates of the matching patterns described above; and we estimate their variances by the Huber-EickerWhite formula:

$$
\hat{V}_{I c}^{m}(J, L)=\hat{k}_{I c}^{m} \widehat{\operatorname{Pr}}(J \mid I, c)(\mathbb{1}(J=L)-\widehat{\operatorname{Pr}}(L \mid I, c))
$$

and

$$
\hat{V}_{J c}^{w}(I, K)=\hat{k}_{J c}^{f} \widehat{\operatorname{Pr}}(I \mid J, c)(\mathbb{1}(I=K)-\widehat{\operatorname{Pr}}(K \mid J, c))
$$

where the factors $\hat{k}$ account for the sampling weights: for instance,

$$
\hat{k}_{I c}^{m}=\frac{\sum_{h} w_{h}^{2} \mathbb{1}(h \supseteq I, c)}{\left(\sum_{h} w_{h} \mathbb{1}(h \supseteq I, c)\right)^{2}} .
$$


C Figures and Tables 


\begin{tabular}{|l|c|c|c|c|c|c|}
\hline & \multicolumn{2}{|c|}{ USA } & \multicolumn{2}{c|}{ Canada } & \multicolumn{2}{c|}{ UK } \\
\hline Year of survey & 1975 & 2003 & 1971 & 1998 & 1975 & 2000 \\
\hline \multicolumn{7}{|c|}{ Domestic chores } \\
\hline Married men, child 5-17 & 1.18 & 1.52 & 1.56 & 1.63 & 0.97 & 1.70 \\
\hline Married women, child 5-17 & 3.63 & 2.83 & 4.55 & 3.29 & 4.01 & 3.37 \\
\hline Married men, child <5 & 1.10 & 1.38 & 1.83 & 1.66 & 0.90 & 1.42 \\
\hline Married women, child <5 & 3.67 & 2.64 & 4.79 & 3.03 & 4.13 & 3.03 \\
\hline \multicolumn{8}{|c|}{ Child care } \\
\hline Married men, child 5-17 & 0.20 & 0.57 & 0.14 & 0.41 & 0.06 & 0.26 \\
\hline Married women, child 5-17 & 0.65 & 1.13 & 0.64 & 0.77 & 0.30 & 0.58 \\
\hline Married men, child <5 & 0.40 & 1.24 & 1.21 & 1.47 & 0.28 & 1.04 \\
\hline Married women, child $<5$ & 1.63 & 2.67 & 2.16 & 2.97 & 1.28 & 2.57 \\
\hline \hline
\end{tabular}

Table 1: Time use (Source: Browning, Chiappori and Weiss 2015) 


\begin{tabular}{|ll|r|}
\hline \multicolumn{2}{|l|}{ Education levels } & $D^{I I, K K} \boldsymbol{b}$ \\
\hline$I$ & $K$ & Value \\
& & (Standard error) \\
\hline 1 & 2 & 0.91 \\
& & $(0.12)$ \\
\hline 1 & 3 & 0.57 \\
& & $(0.18)$ \\
\hline 1 & 4 & 0.11 \\
& & $(0.27)$ \\
\hline 1 & 5 & 0.51 \\
& & $(0.40)$ \\
\hline 2 & 3 & -0.09 \\
& & $(0.05)$ \\
\hline 2 & 4 & 0.30 \\
& & $(0.07)$ \\
\hline 2 & 5 & 0.39 \\
& & $(0.13)$ \\
\hline 3 & 4 & 0.35 \\
& & $(0.08)$ \\
\hline 3 & 5 & 0.70 \\
& & $(0.10)$ \\
\hline 4 & 5 & 0.37 \\
& & $(0.08)$ \\
\hline \hline
\end{tabular}

Table 2: Estimated trends in complementarities for whites 


\begin{tabular}{|l|rrr|rrr|}
\hline & \multicolumn{4}{|c}{ Men } & \multicolumn{3}{c|}{ Women } \\
\cline { 2 - 7 } & Early & Late & Change & Early & Late & Change \\
\hline HS Dropouts & 0.85 & 0.48 & -0.37 & 0.86 & 0.61 & -0.25 \\
HS Graduates & 0.90 & 0.62 & -0.27 & 0.92 & 0.77 & -0.15 \\
Some College & 0.90 & 0.67 & -0.22 & 0.90 & 0.78 & -0.12 \\
College & 0.89 & 0.75 & -0.13 & 0.88 & 0.81 & -0.07 \\
College-plus & 0.90 & 0.80 & -0.10 & 0.79 & 0.77 & -0.03 \\
\hline
\end{tabular}

Table 3: Probabilities of marriage 


\begin{tabular}{|l|ccccc||}
\hline Wife $\rightarrow$ & HSD & HSG & SC & CG & CG + \\
\hline Husband $\downarrow$ & \multicolumn{5}{|c||}{ Early Cohorts } \\
\hline HSD & 0.37 & 0.47 & 0.14 & 0.02 & 0.01 \\
HSG & 0.09 & 0.65 & 0.20 & 0.04 & 0.02 \\
SC & 0.05 & 0.41 & 0.41 & 0.09 & 0.05 \\
CG & 0.01 & 0.21 & 0.33 & 0.32 & 0.13 \\
CG + & 0.01 & 0.10 & 0.24 & 0.34 & 0.32 \\
\hline Husband $\downarrow$ & \multicolumn{5}{|c||}{ Late Cohorts } \\
\hline HSD & 0.29 & 0.39 & 0.26 & 0.05 & 0.02 \\
HSG & 0.04 & 0.46 & 0.33 & 0.12 & 0.04 \\
SC & 0.01 & 0.20 & 0.46 & 0.23 & 0.09 \\
CG & 0.00 & 0.07 & 0.21 & 0.52 & 0.20 \\
CG + & 0.00 & 0.03 & 0.12 & 0.42 & 0.42 \\
\hline Husband $\downarrow$ & \multicolumn{5}{|c||}{ Change } \\
\hline HSD & -0.08 & -0.08 & 0.12 & 0.03 & 0.01 \\
HSG & -0.05 & -0.18 & 0.13 & 0.08 & 0.02 \\
SC & -0.03 & -0.21 & 0.06 & 0.14 & 0.04 \\
CG & -0.01 & -0.14 & -0.12 & 0.20 & 0.07 \\
CG + & -0.00 & -0.07 & -0.11 & 0.08 & 0.11 \\
\hline
\end{tabular}

Table 4: Partners of men (men in rows, women in columns) 


\begin{tabular}{|l|ccccc|}
\hline Husband $\rightarrow$ & HSD & HSG & SC & CG & CG + \\
\hline Wife $\downarrow$ & \multicolumn{5}{|c|}{ Early Cohorts } \\
\hline HSD & 0.48 & 0.34 & 0.14 & 0.02 & 0.02 \\
HSG & 0.13 & 0.48 & 0.25 & 0.10 & 0.05 \\
SC & 0.05 & 0.21 & 0.37 & 0.21 & 0.16 \\
CG & 0.01 & 0.07 & 0.14 & 0.36 & 0.41 \\
CG + & 0.01 & 0.06 & 0.11 & 0.22 & 0.60 \\
\hline Wife $\downarrow$ & \multicolumn{5}{|c|}{ Late Cohorts } \\
\hline HSD & 0.42 & 0.37 & 0.15 & 0.04 & 0.02 \\
HSG & 0.09 & 0.53 & 0.26 & 0.10 & 0.03 \\
SC & 0.04 & 0.26 & 0.41 & 0.20 & 0.08 \\
CG & 0.01 & 0.10 & 0.19 & 0.46 & 0.24 \\
CG+ & 0.01 & 0.06 & 0.14 & 0.34 & 0.45 \\
\hline Wife $\downarrow$ & -0.06 & 0.03 & 0.01 & 0.02 & -0.00 \\
\hline HSD & -0.01 & 0.00 & -0.02 \\
HSG & -0.04 & 0.05 & 0.01 & 0.01 & -0.08 \\
SC & -0.01 & 0.06 & 0.05 & -0.01 \\
CG & -0.00 & 0.02 & 0.05 & 0.10 & -0.17 \\
CG + & -0.00 & 0.01 & 0.03 & 0.12 & -0.15 \\
\hline
\end{tabular}

Table 5: Partners of women (women in rows, men in columns) 


\begin{tabular}{|l|rrrrr||}
\hline Wife $\rightarrow$ & HSD & HSG & \multicolumn{1}{c|}{ SC } & CG & CG+ \\
\hline Husband $\downarrow$ & \multicolumn{5}{|c|}{ Early Cohorts } \\
\hline HSD & -6.48 & -7.55 & -9.91 & -13.24 & -14.17 \\
HSG & -7.76 & -5.36 & -7.54 & -9.95 & -11.13 \\
SC & -9.90 & -6.90 & -6.60 & -8.86 & -9.95 \\
CG & -12.65 & -8.25 & -7.11 & -6.59 & -8.04 \\
CG+ & -13.67 & -9.46 & -7.48 & -6.14 & -6.04 \\
\hline Husband $\downarrow$ & \multicolumn{5}{|c||}{ Late Cohorts } \\
\hline HSD & -7.69 & -8.92 & -10.27 & -13.10 & -14.81 \\
HSG & -9.23 & -6.18 & -7.30 & -9.09 & -10.56 \\
SC & -11.47 & -8.10 & -6.85 & -8.08 & -9.41 \\
CG & -13.44 & -9.61 & -7.84 & -6.01 & -7.38 \\
CG+ & -14.89 & -11.42 & -9.12 & -6.72 & -6.23 \\
\hline Husband $\downarrow$ & \multicolumn{6}{|c}{ Change (positive values in bold) } \\
\hline HSD & -1.21 & -1.37 & -0.36 & $\mathbf{0 . 1 4}$ & -0.64 \\
HSG & -1.47 & -0.82 & $\mathbf{0 . 2 3}$ & $\mathbf{0 . 8 6}$ & $\mathbf{0 . 5 7}$ \\
SC & -1.58 & -1.20 & -0.25 & $\mathbf{0 . 7 8}$ & $\mathbf{0 . 5 4}$ \\
CG & -0.80 & -1.36 & -0.74 & $\mathbf{0 . 5 8}$ & $\mathbf{0 . 6 5}$ \\
CG+ & -1.22 & -1.95 & -1.63 & -0.58 & -0.19 \\
\hline
\end{tabular}

Table 6: Joint surplus (men in rows, women in columns) 


\begin{tabular}{|l|ccccc|}
\hline Wife $\rightarrow$ & HSD & HSG & SC & CG & CG+ \\
\hline Husband $\downarrow$ & \multicolumn{5}{|c|}{ Early Cohorts } \\
\hline HSD & 0.51 & 0.57 & 0.55 & 0.53 & 0.45 \\
HSG & 0.47 & 0.53 & 0.51 & 0.48 & 0.41 \\
SC & 0.46 & 0.53 & 0.51 & 0.48 & 0.41 \\
CG & 0.48 & 0.54 & 0.52 & 0.49 & 0.42 \\
CG+ & 0.46 & 0.53 & 0.51 & 0.48 & 0.41 \\
\hline \hline Husband $\downarrow$ & \multicolumn{5}{|c|}{ Late Cohorts } \\
\hline HSD & 0.59 & 0.69 & 0.70 & 0.72 & 0.69 \\
HSG & 0.49 & 0.60 & 0.61 & 0.63 & 0.60 \\
SC & 0.46 & 0.56 & 0.57 & 0.59 & 0.56 \\
CG & 0.40 & 0.51 & 0.52 & 0.54 & 0.51 \\
CG+ & 0.37 & 0.48 & 0.49 & 0.51 & 0.48 \\
\hline Husband $\downarrow$ & Change (positive values in bold) \\
\hline HSD & $\mathbf{0 . 0 8}$ & $\mathbf{0 . 1 2}$ & $\mathbf{0 . 1 5}$ & $\mathbf{0 . 1 9}$ & $\mathbf{0 . 2 4}$ \\
HSG & $\mathbf{0 . 0 3}$ & $\mathbf{0 . 0 7}$ & $\mathbf{0 . 1 0}$ & $\mathbf{0 . 1 4}$ & $\mathbf{0 . 1 9}$ \\
SC & -0.01 & $\mathbf{0 . 0 4}$ & $\mathbf{0 . 0 7}$ & $\mathbf{0 . 1 1}$ & $\mathbf{0 . 1 6}$ \\
CG & -0.07 & -0.03 & 0.00 & $\mathbf{0 . 0 5}$ & $\mathbf{0 . 0 9}$ \\
CG+ & -0.09 & -0.05 & -0.02 & $\mathbf{0 . 0 3}$ & $\mathbf{0 . 0 7}$ \\
\hline
\end{tabular}

Table 7: Surplus share of wife (men in rows, women in columns) 


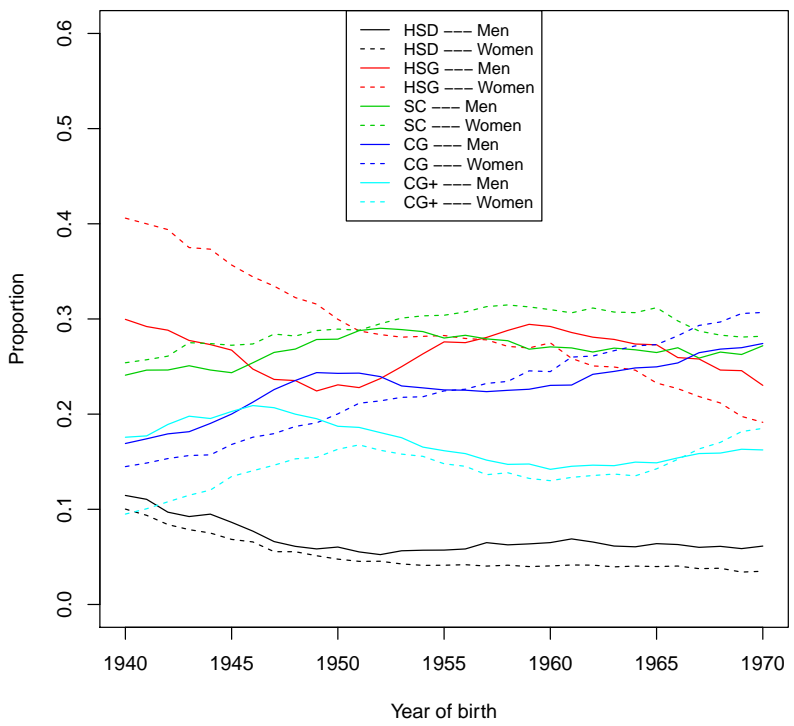

Figure 1: Educations of white men and women 


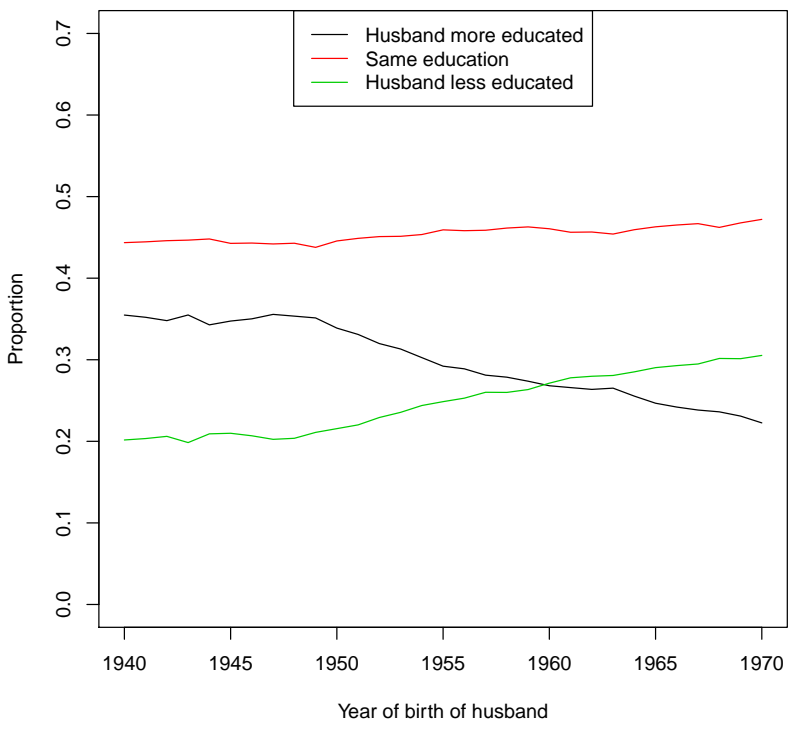

Figure 2: Comparing partners in white couples 

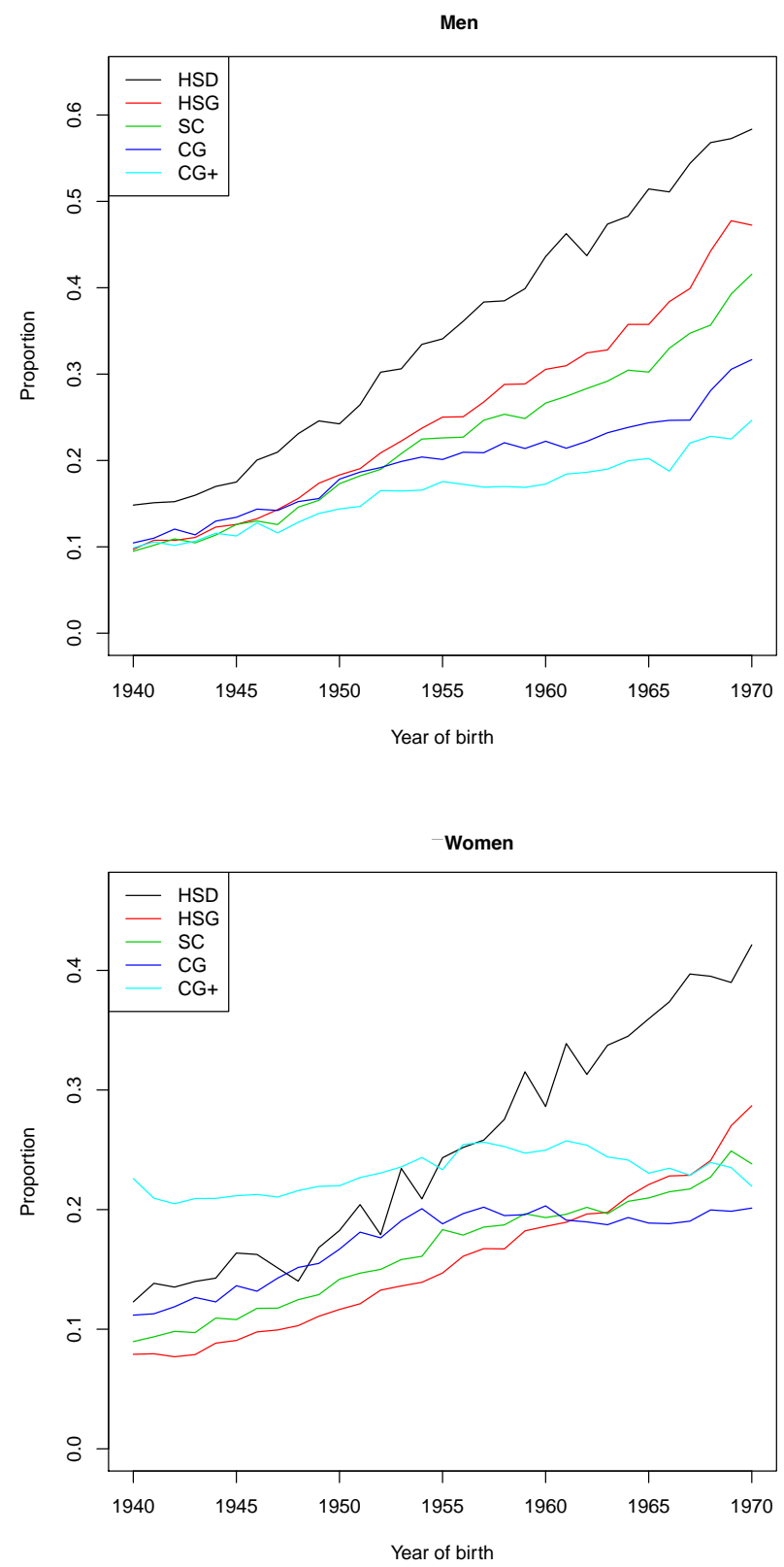

Figure 3: Never married white men and women 


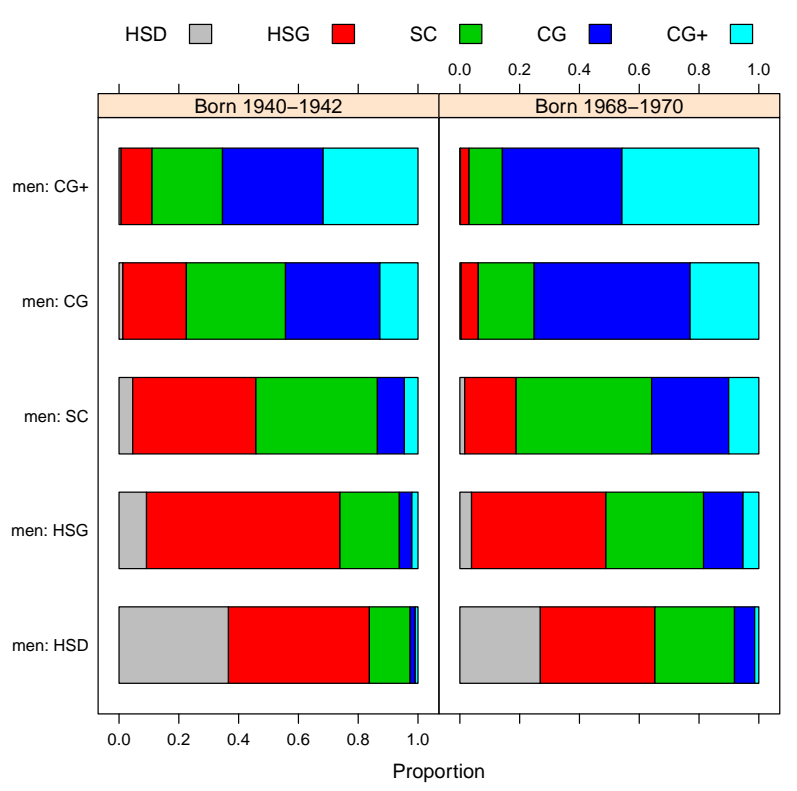

Figure 4: Marriage patterns of white men who marry 


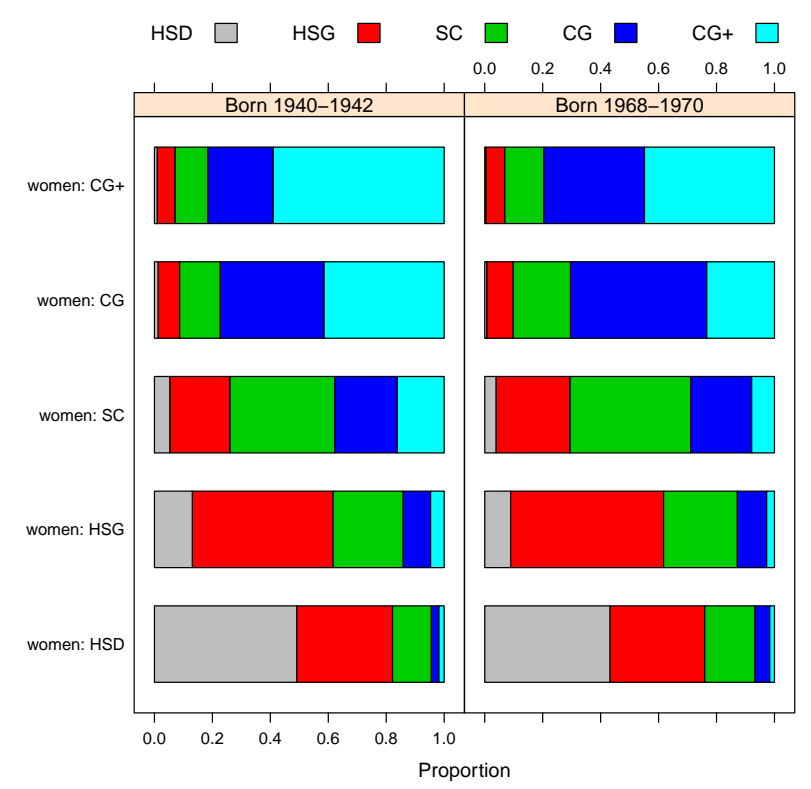

Figure 5: Marriage patterns of white women who marry 


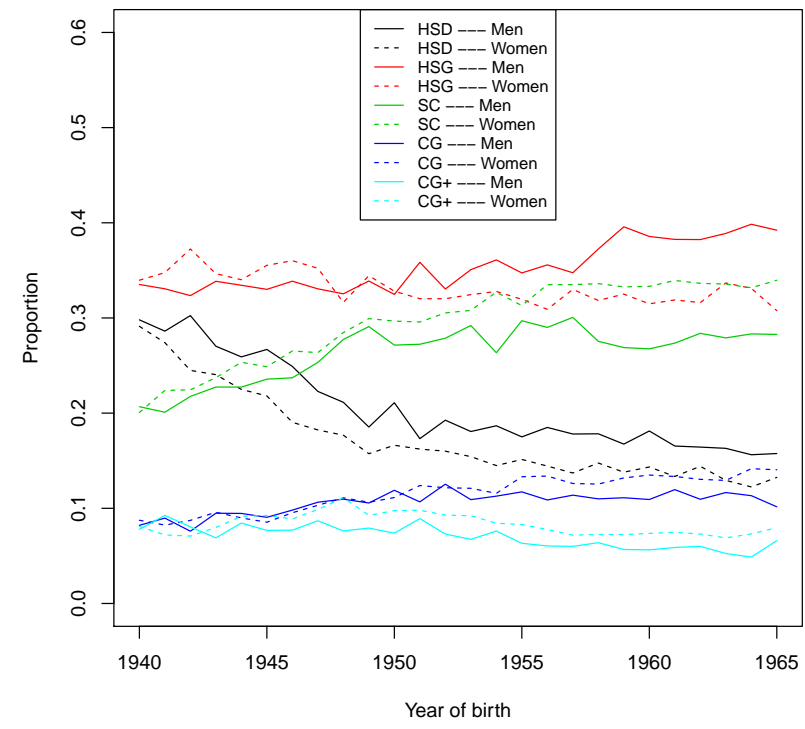

Figure 6: Educations of black men and women 


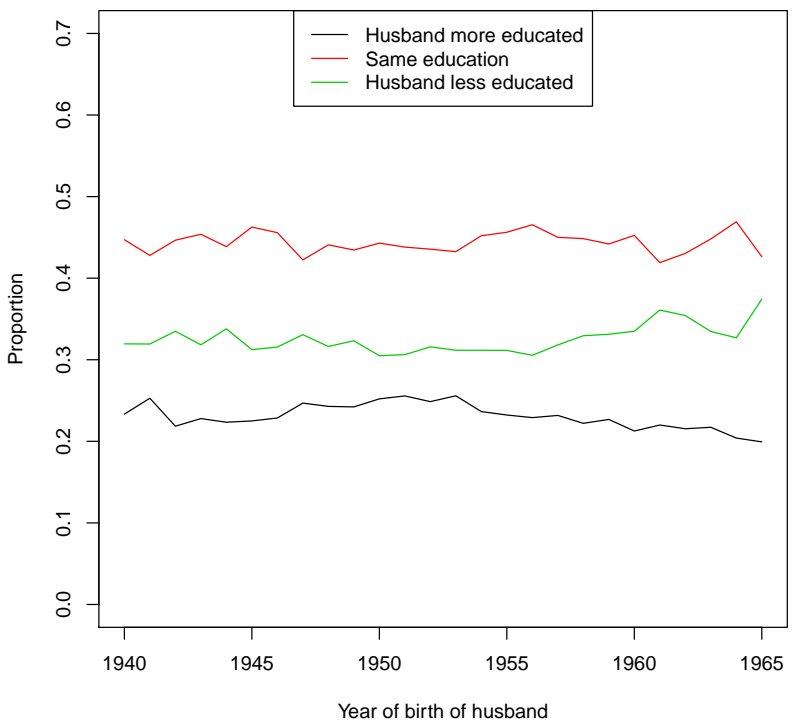

Figure 7: Comparing partners in black couples 

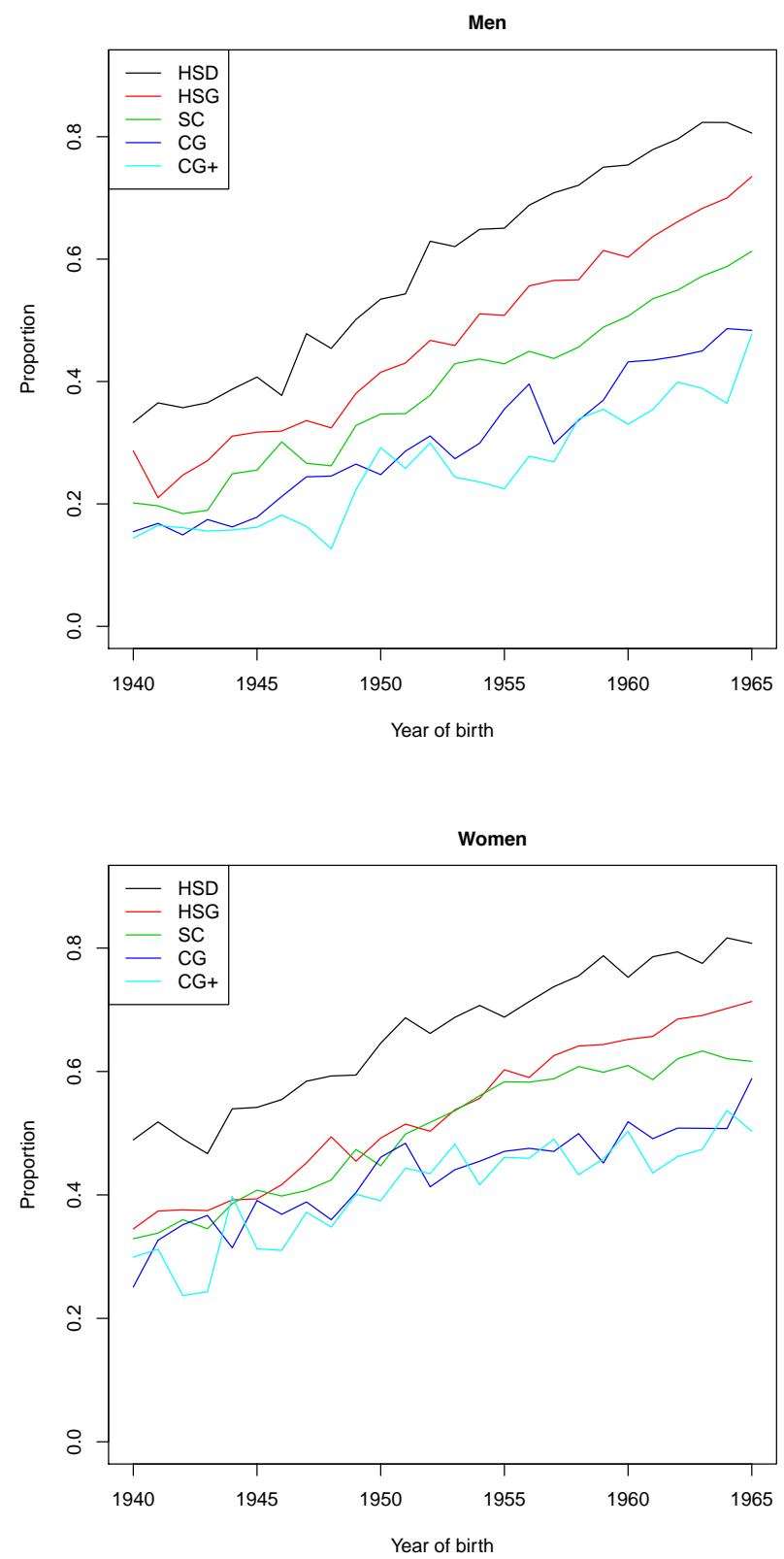

Figure 8: Never married black men and women 


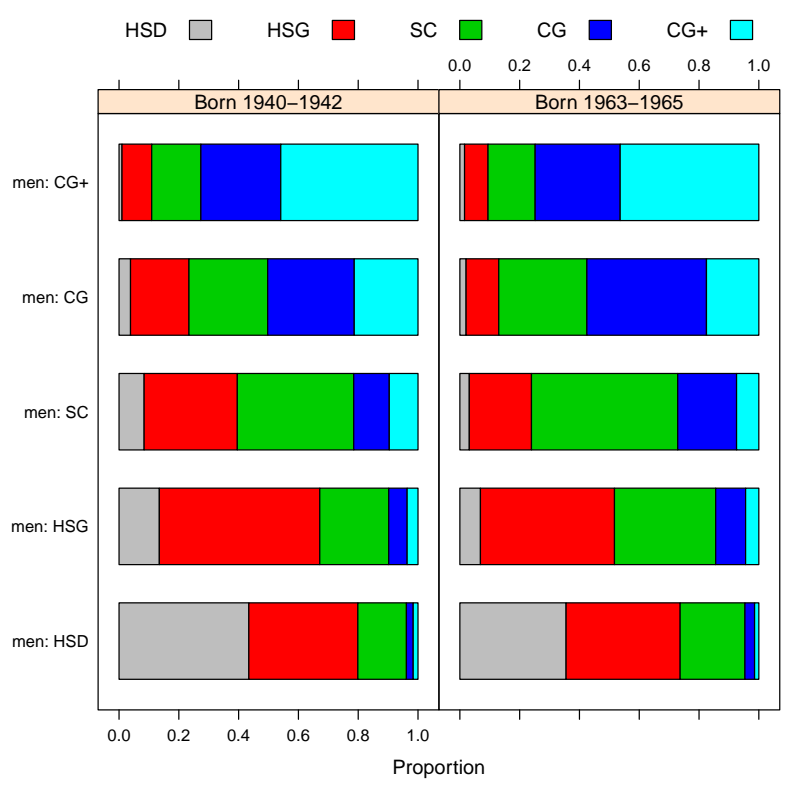

Figure 9: Marriage patterns of black men who marry 


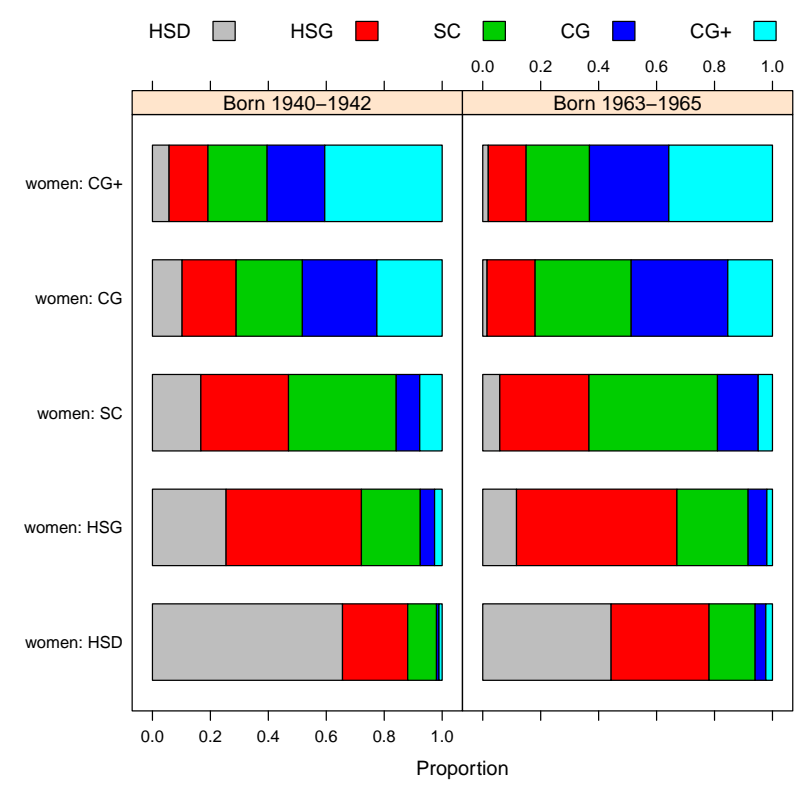

Figure 10: Marriage patterns of black women who marry 

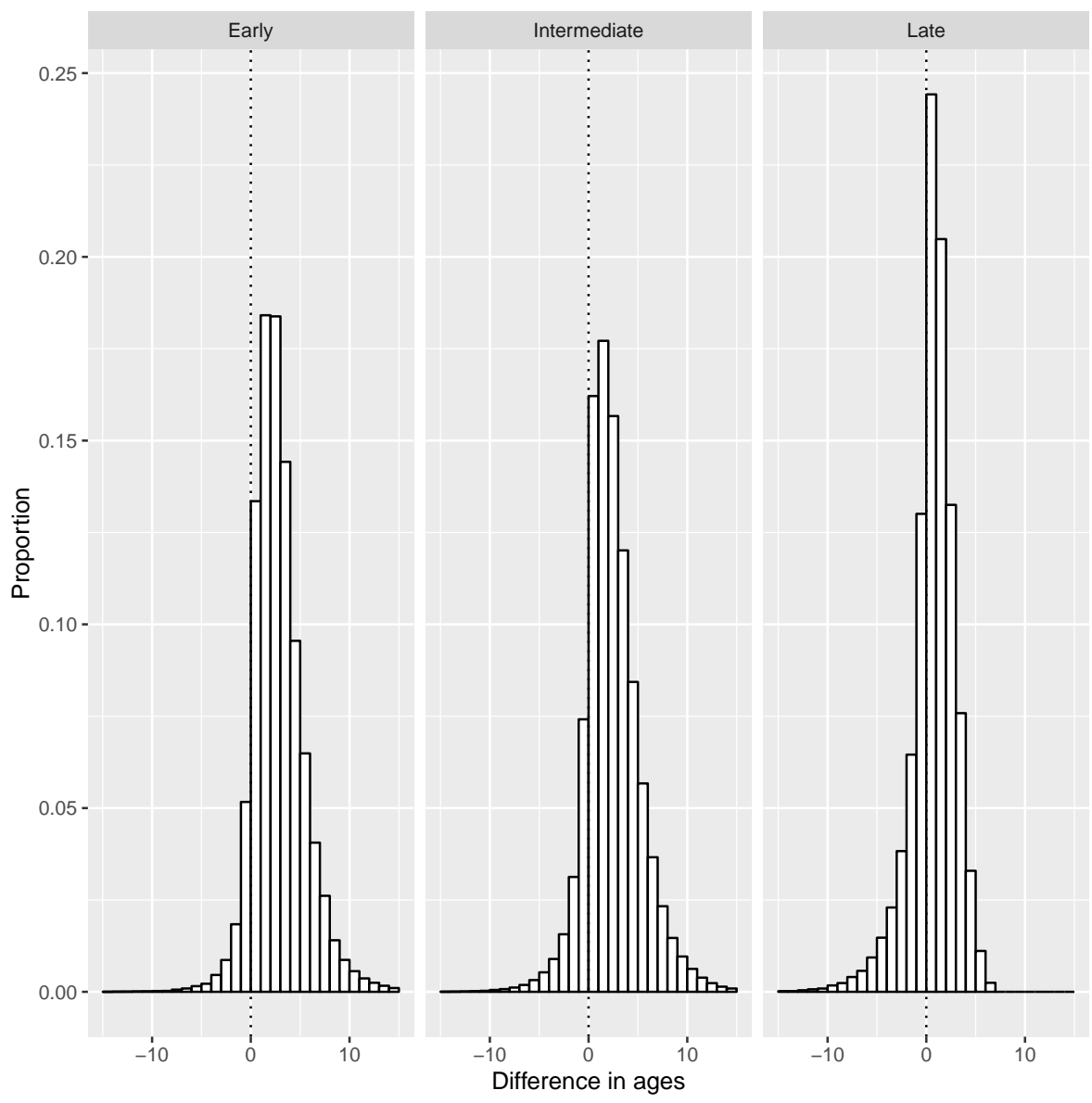

Figure 11: Difference in ages of partners (husband-wife) in white couples 

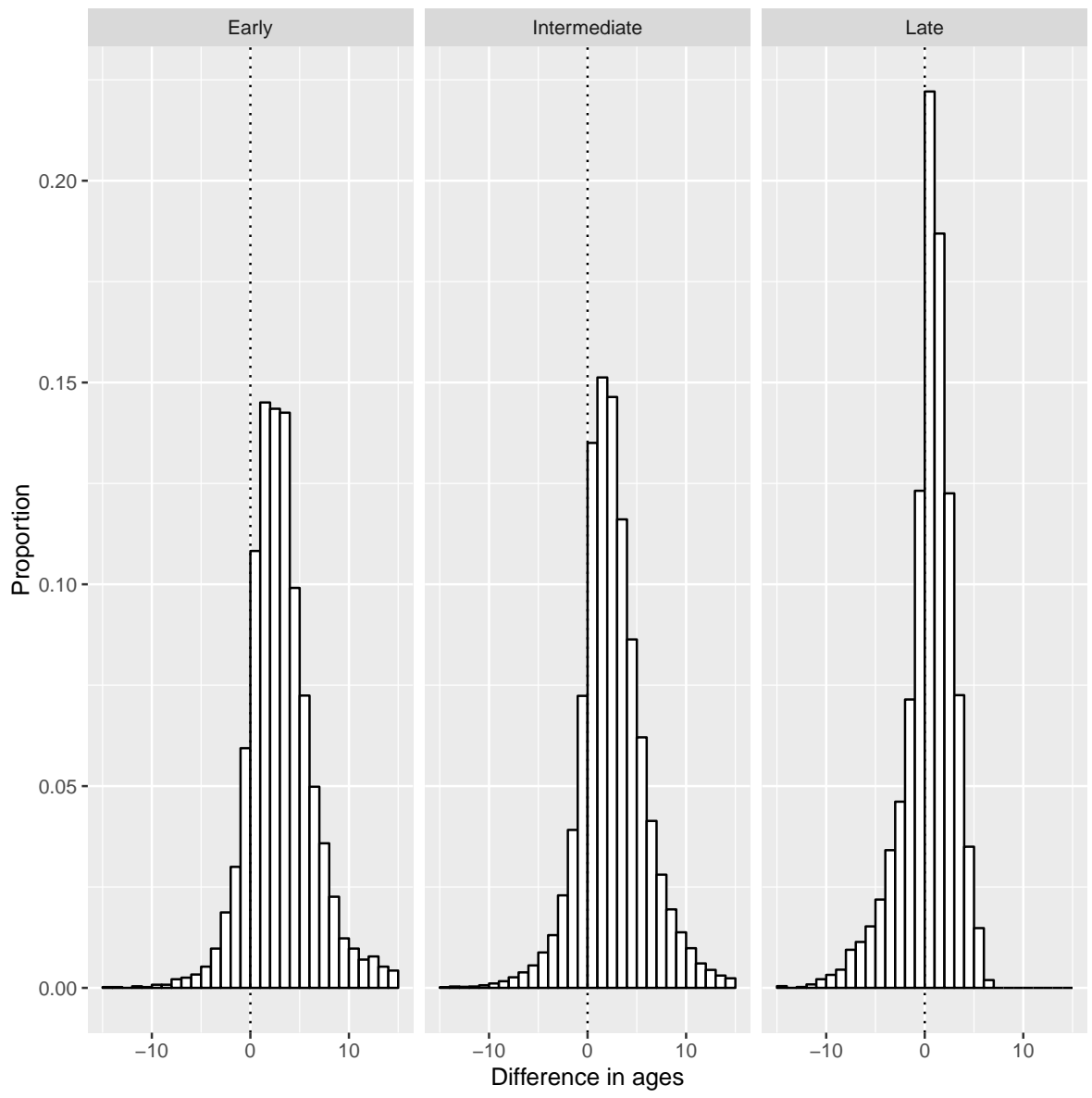

Figure 12: Difference in ages of partners (husband-wife) in black couples 


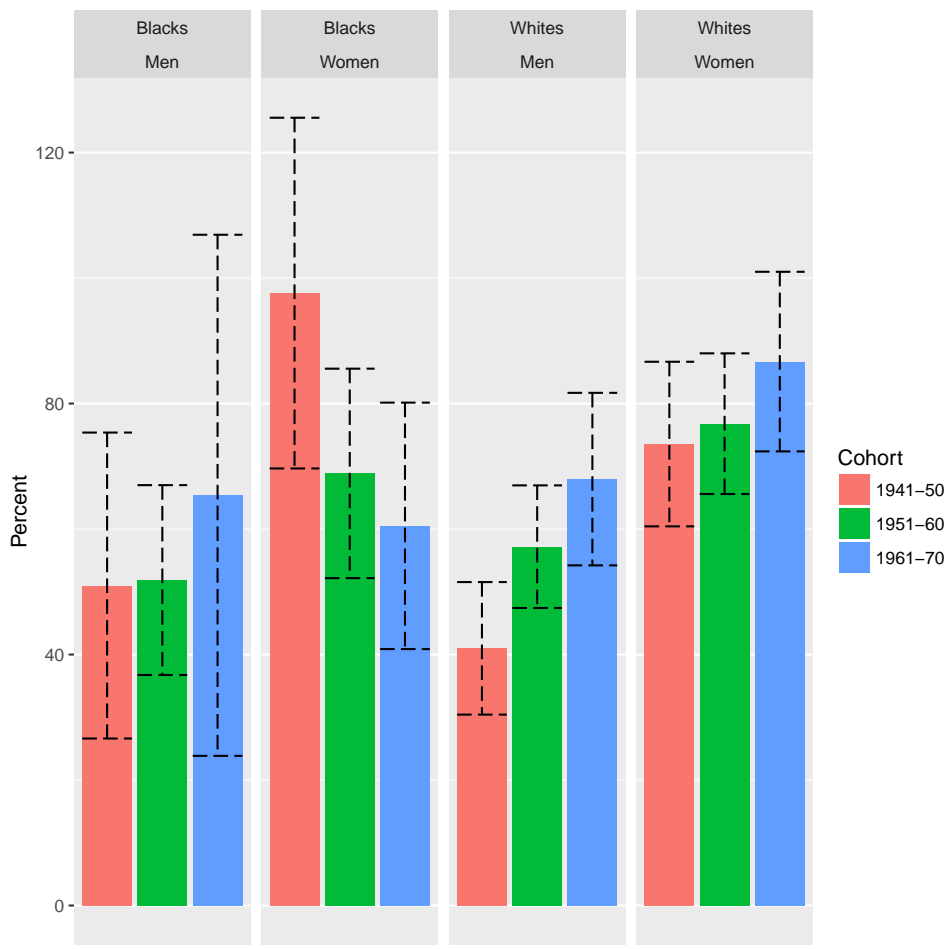

The college premium is defined as the percentage difference between hourly wages after a 4-year college degree and after high-schol graduation for an individual of age 35. The error bars reresent 95\% confidence intervals. Source: PSID, with help from George-Levi Gayle, Limor Golan, and Andres Hincapie.

Figure 13: Estimated labor market college premia 


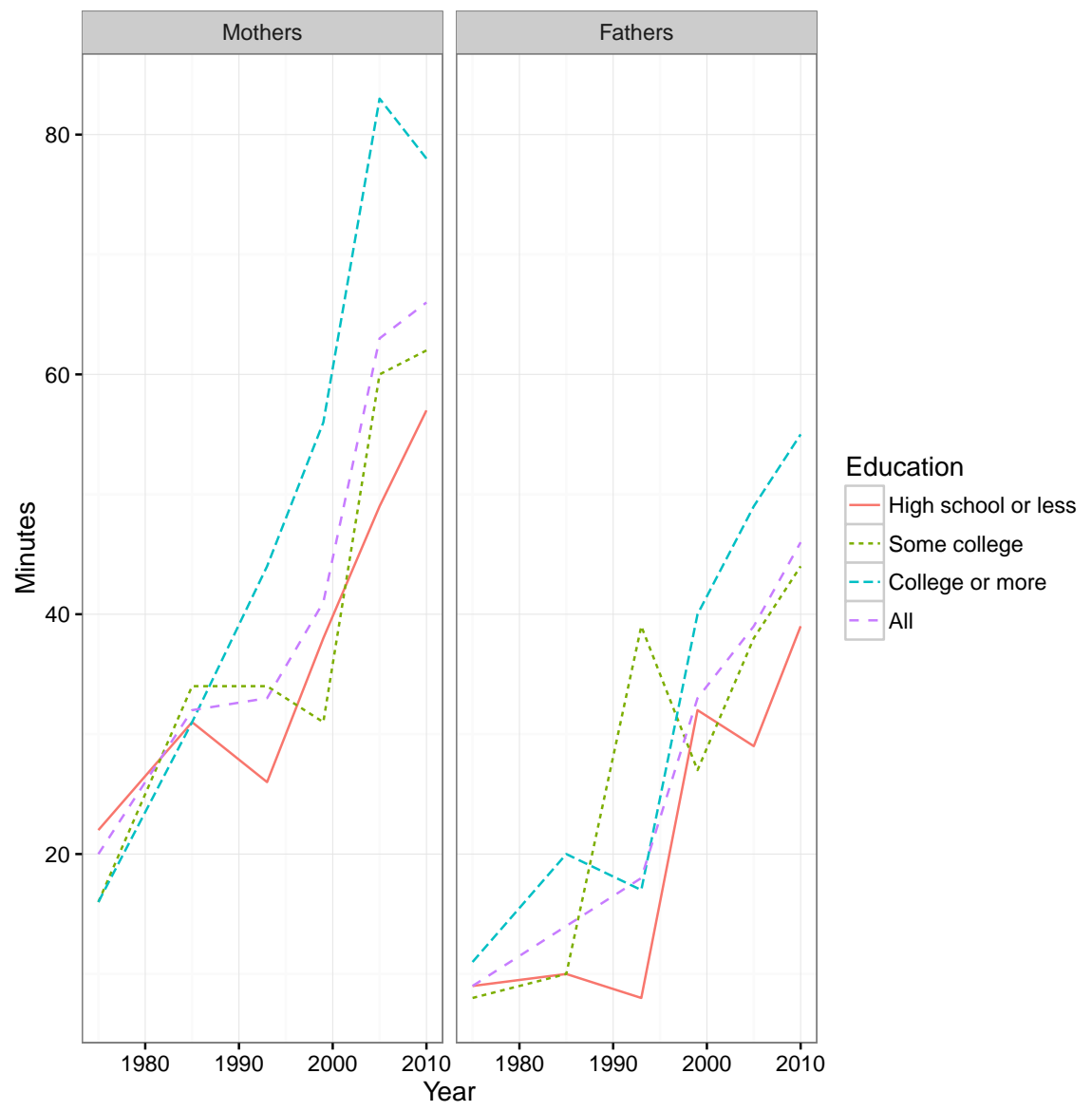

Figure 14: Average minutes spent daily in developmental child care, US (Source: Altintas 2016) 


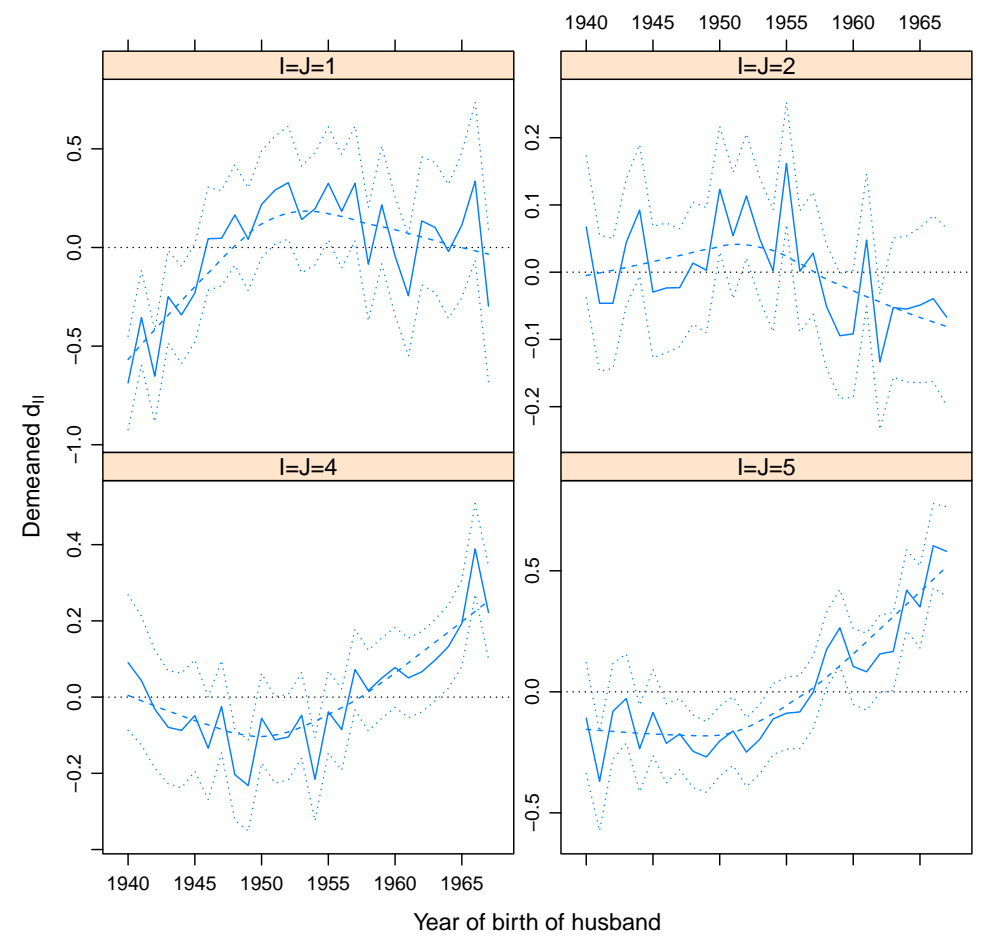

Figure 15: Testing the benchmark model for whites 


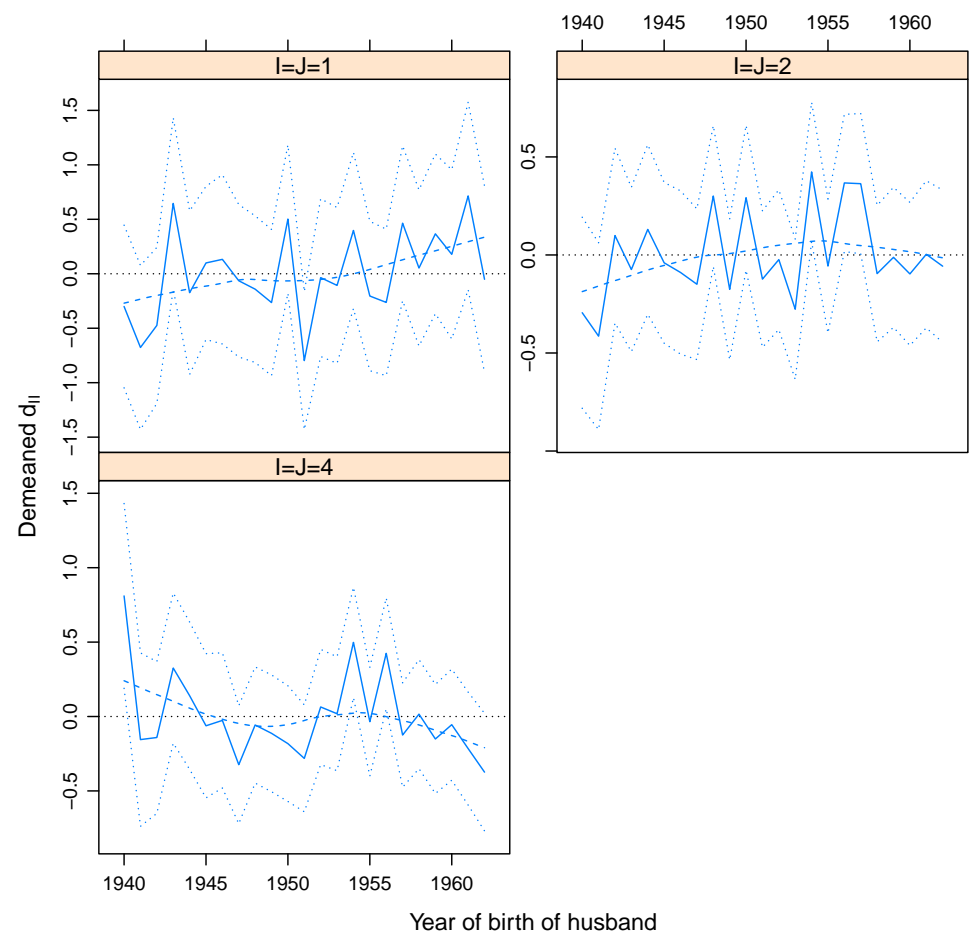

Figure 16: Testing the benchmark model for blacks 


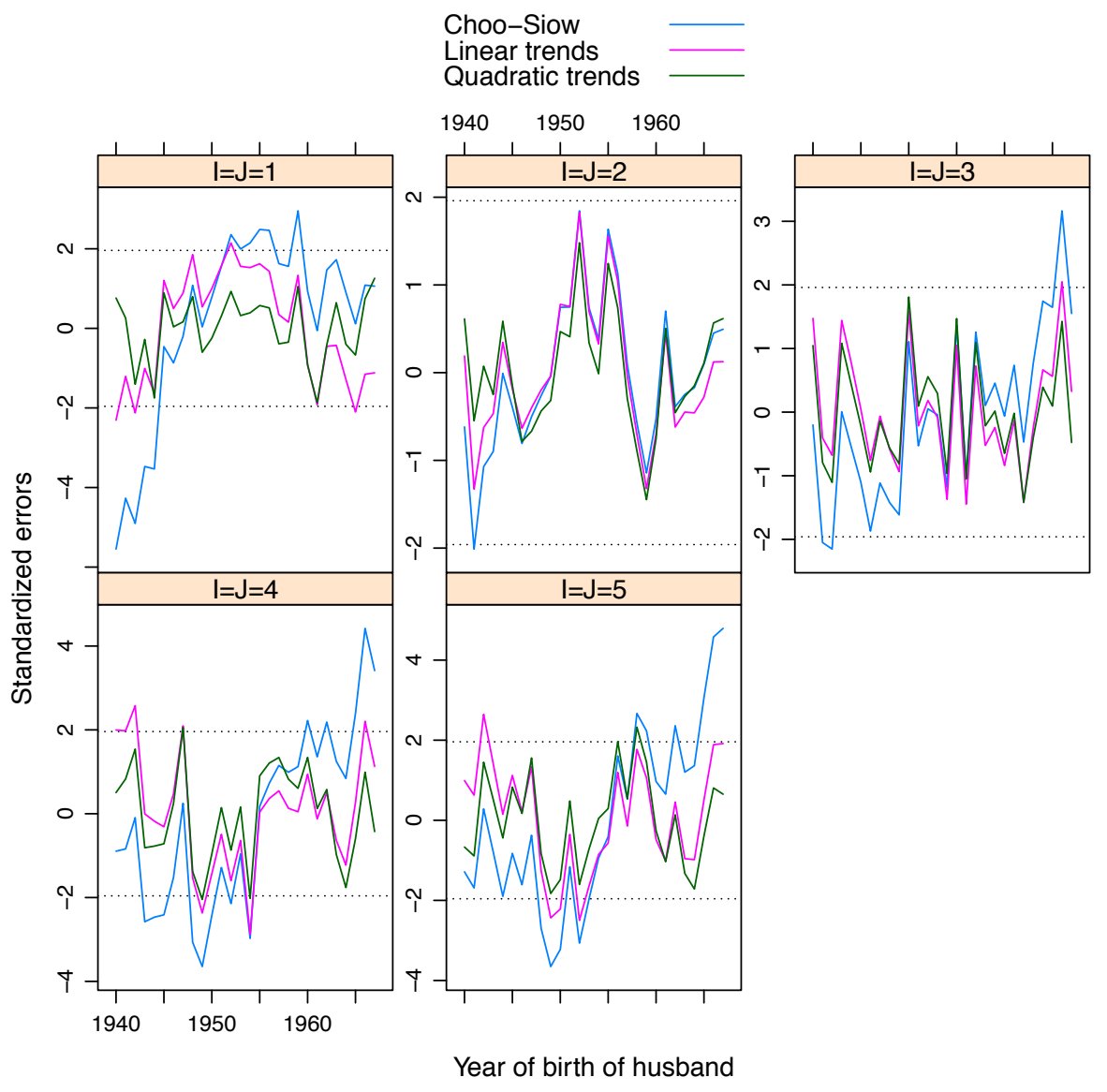

Figure 17: Testing various models for whites 


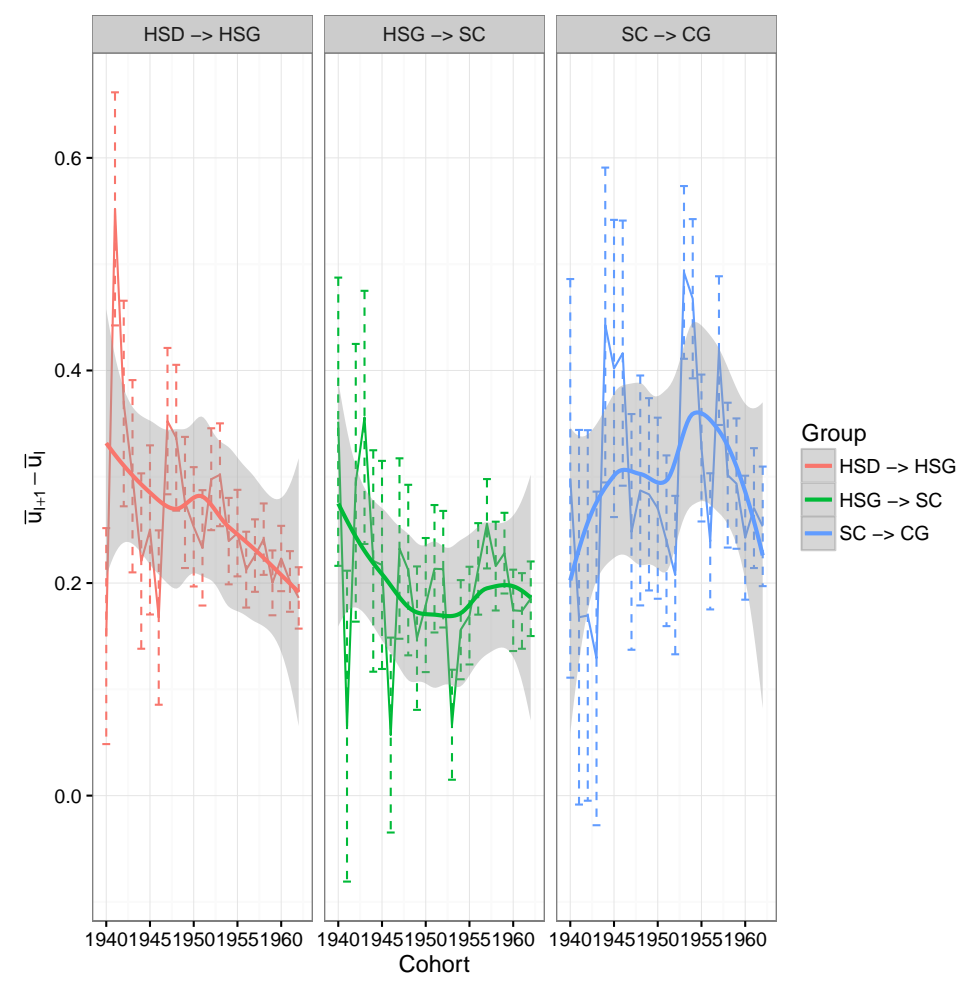

Figure 18: Education premia of black men (one standard error confidence bands) 


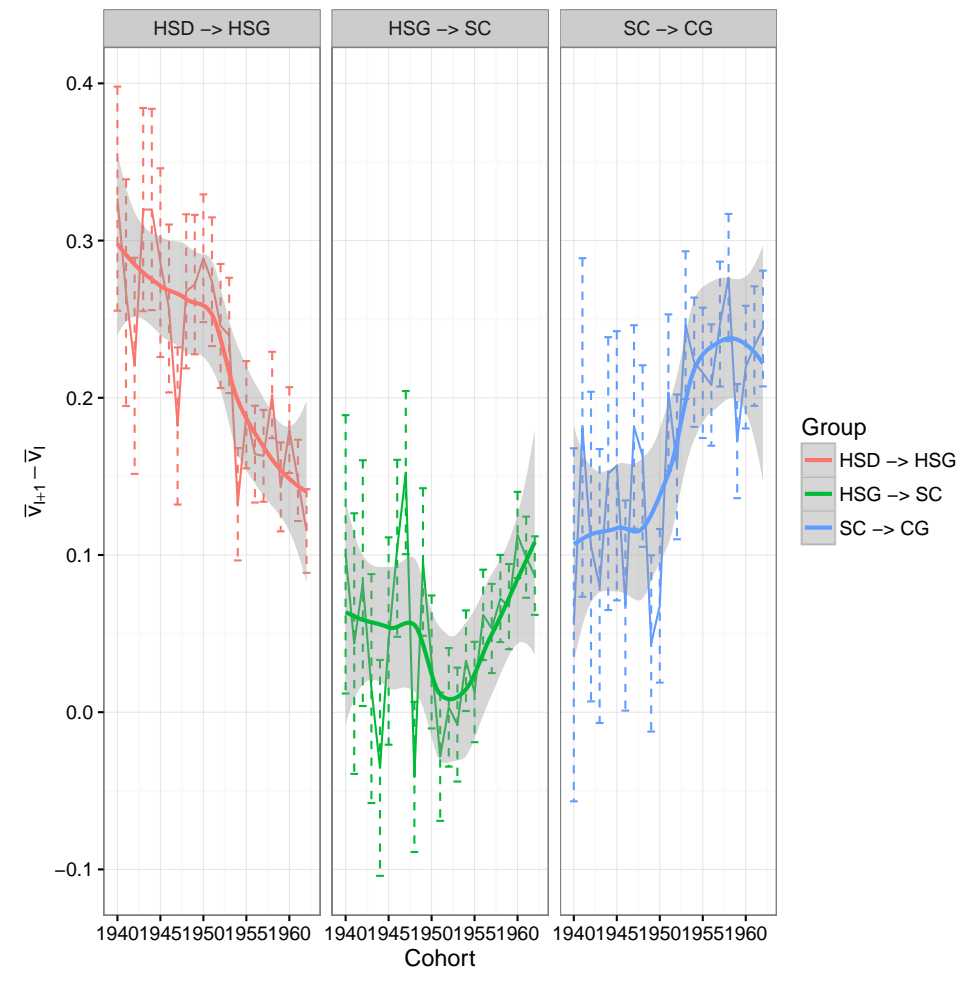

Figure 19: Education premia of black women (one standard error confidence bands) 


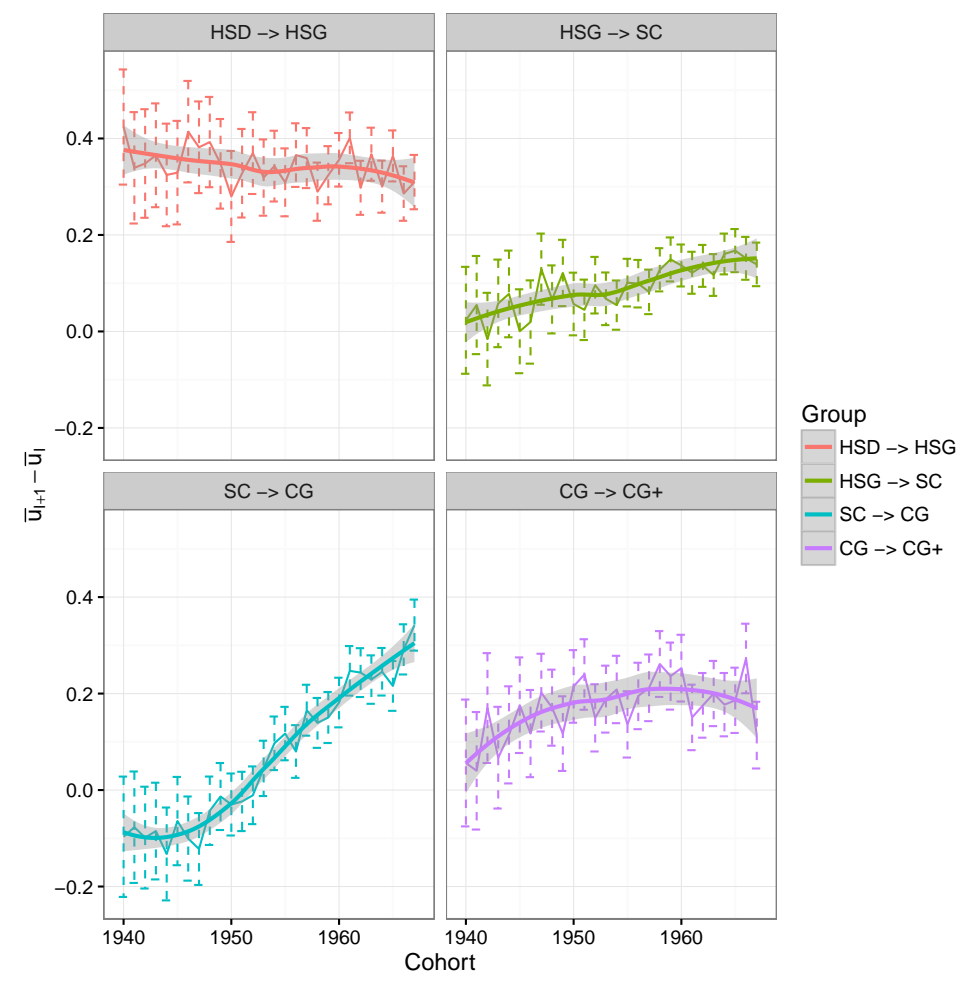

Figure 20: Education premia of white men 


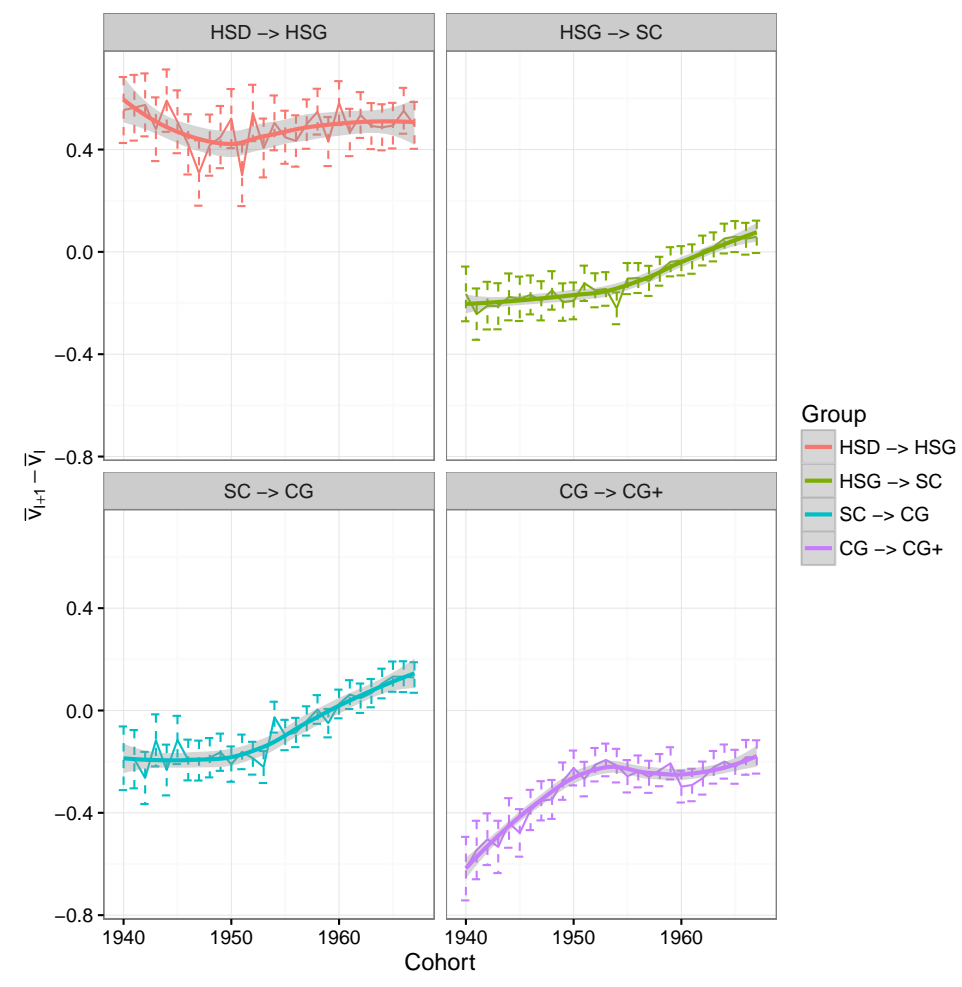

Figure 21: Education premia of white women 


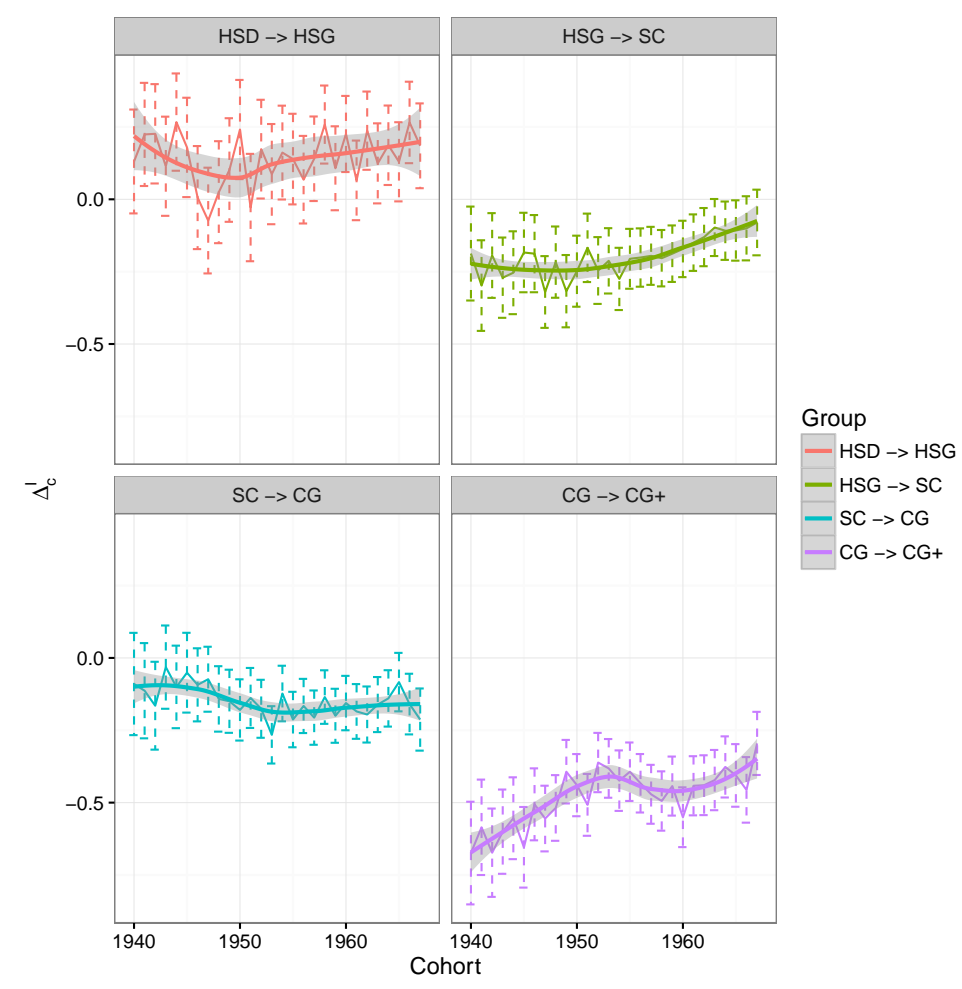

Figure 22: Estimated excess premia for white women 